\title{
The Political Economy of Employment Protection ${ }^{1}$
}

\author{
Gilles Saint-Paul \\ Universitat Pompeu Fabra and CEPR
}

January 27, 1999

\footnotetext{
${ }^{1}$ The author thanks participants at seminars at CEMFI, Madrid, the Bank of Italy, the Federal Reserve Bank of Saint-Louis, the Séminaire René Roy, Paris, for helpful comments and suggestions, as well as the Bank of Spain and CREI for financial support.
} 


\section{Introduction}

Many economists believe that labor market rigidities are responsible for the high level of unemployment in Europe. Such rigidities include, among other things, high minimum wages, generous unemployment benefits, and severe employment protection legislation. Many policy institutions such as the OECD or the IMF typically advocate a reduction or removal of these rigidities as a cure for unemployment. However, such reforms have rarely been seen in practice, and politicians that tried to implement them have often faced considerable political opposition.

This suggests that policy prescriptions could be improved if one approached the issue from a political economy perspective, understanding who gains and who loses from a given institution and why it exists in the first place.

In this paper, we analyze the political economy of employment protection legislation, that we model as a tax on layoffs (or 'firing cost'). Our key insight is that the support for such legislation comes from the existence of rents enjoyed by incumbent employees over their alternative wages, i.e. from other imperfections in the labor market that prevent wages from adjusting to their market-clearing level. ${ }^{1}$ Furthermore, a lot of attention is paid to the role of firing costs in the growth process when it is associated with obsolescence. In our vintage capital model each match gradually gets obsolete (because its productivity fails to catch up with the latest technology) until it is destroyed, in which case the worker becomes unemployed. Firing costs alter the obsolescence decision by increasing the duration of matches.

\footnotetext{
${ }^{1}$ This rent is itself influenced by institutions but we take it as given and study its impact on the equilibrium level of dismissal cost.
} 
In voting in favor of employment protection, incumbent employees trade off lower wages (because employment protection maintains workers in less productive activities) against longer job duration. The support for employment protection will then depend on the value of the latter relative to the cost of the former. We highlight two key determinants of this trade-off: first, the workers' bargaining power, second, the economy's growth rate - more precisely its rate of creative destruction. Let us explain briefly the mechanisms that underlie the effect of these two parameters.

The rent. The value of longer job duration to incumbent workers is proportional to the rent, or equivalently their bargaining power; long job duration would not be valued if the employed were not earning rents above the unemployed. The cost of job loss would then be zero, and so would the support for employment protection. This result tells us that there exists a "complementarity" between firing costs and other labor market rigidities to the extent that the latter increase workers' bargaining power.

One important consequence is the existence of complementarities across policy reforms. A comprehensive labor market reform that attacks those rigidities that increase workers' bargaining power at the same time that it reduces firing costs is more likely to be successful than one that only tackles the latter aspect. (Coe and Snower (1997) analyze the importance of policy complementarities in labor market reforms).

Creative destruction. Firing costs reduce the economy's average productivity by maintaining a fraction of the workforce in vintages that are older than the most up-to-date technology. In equilibrium, this ends up reducing wages and living standards. Now, this effect will be stronger, the greater the productivity gap between old vintages and new vintages, i.e., the greater the growth rate. A higher growth rate consequently reduces the political support for employment protection legislation, because it increases its cost in terms of lower wages. 
My model also sheds light on which workers will support employment protection, and who will be against it. The unemployed typically oppose it since it both reduces their likelihood of finding a job and the average wage that this job will pay. We also show that higher firing costs are typically supported by workers at older plants, while workers are younger plants prefer lower firing costs. The reason is that the gain of higher firing costs in terms of extended job duration is more remote, and therefore more heavily discounted, for the latter than for the former, while both bear similar losses in terms of lower wages. However, this has to be qualified. We show that there exists a group of workers in very old plants (the "lost generation"), who are about to be laid off, and support either an increase in firing costs that postpones this event, or a reduction in firing costs that would increase their job prospects as unemployed. This group may potentially generate Condorcet cycles in the determination of the political equilibrium, although a majority winner over marginal alternatives exists for a wide range of parameter values.

This paper is related to various strands of literature, although to my knowledge it is the first one that analyzes the political economy of employment protection in its connections with labor market imperfections and economic growth. In Saint-Paul (1993), I deal with a specific aspect of that issue, namely how reducing firing costs by means of a two-tier system may be politically feasible, using a model with fixed wages and no growth. Recent work on the political economy of labor market institutions includes Robinson (1997), Acemoglu and Robinson (1998), Hassler et al. (1998), and Grüner (1998), but is concerned with different aspects. Robinson (1997) mainly deals with the political economy of workers' bargaining power, rather than employment protection, in the context of a totally different model. Acemoglu and Robinson (1998) develop a theory of how redistribution may be inefficient. Hassler et al. (1998), following Wright (1986), analyze the political determination of unemployment benefits. Grüner (1998) deals with the important problem 
of why side-transfers may not be feasible in order to buy out losers. Related work also include Krusell and Rios-Rull (1994), who study the political support for technology adoption and its implications for growth; here, as in their model, firing costs somewhat reduce the pace of technology adoption by preventing resources from being relocated from old to new technologies. But their focus is entirely different as they do not study labor market institutions and work with perfect labor markets.

The economic side of the model is more in line with the existing literature. I use the vintage capital model of Johansen (1959), that Caballero and Hammour $(1994,1996)$ also use to analyze the efficiency of job creation and job destruction over the business cycle. Such model is not generally used to study the effects of firing costs, but our results have the same flavor as the ones obtained with other types of models, such as Bentolila and Bertola (1990), Bentolila and Saint-Paul (1994), or, Hopenhayn and Rogerson (1993) in a general equilibrium context and Bertola (1994) in a growth context. Finally, the destructive aspects of growth and their potential consequences on unemployment encountered by Aghion and Howitt (1994) are also present in my model.

The paper is organized as follows: in section 2 we spell out the assumptions of our vintage model. In section 3 we study the properties of the model's solution. In section 4 we allow people to vote on the degree on employment protection and discuss the properties of the political equilibrium. 


\section{The model}

We consider a growth model with different vintages of capital ${ }^{2}$ At any point in time $t$ there is a state of the art technology which allows to produce $a_{t}$ units of output with 1 unit of labor. $a_{t}$ is assumed to grow at a constant exogenous rate $g$, so that $a_{t}=a_{0} e^{g t}$. There is free entry of firms (considered as hiring a single worker) in the state of the art technology; but once a firm has entered it cannot upgrade and is stuck with the level prevailing at the time of entry.

Exit is costly so that in order to close at time $T$ the firm pay a "firing cost" in terms of output, equal to $F a_{T}$. Dependence on the productivity trend implies that the firing cost grows at the same rate as the rest of the economy, and therefore does not become negligible relative to the surplus of a match. We assume that this firing cost is wasted. ${ }^{3}$ Firms are also hit by random shocks that destroy the match with flow probability $\delta$. A match hit by such shock is forced to split and the firm has to pay a fraction $\rho$ of the firing cost. Thus, in the model there are two sources of job destruction: economic obsolescence (the decision of the firm to close because continuing is no longer profitable), associated with firing cost $F a_{T}$, and an exogenous source of turnover associated with firing cost $\rho F a_{T}$. Technically, this shock

\footnotetext{
${ }^{2}$ (Johansen (1959), Caballero and Hammour $\left.(1994,1996)\right)$. Another option would be to rule out growth and assume that once in the market firms suffer shocks to their productivity level and that they can freely enter the market at the maximum possible level, as is done in Mortensen and Pissarides (1994).

${ }^{3}$ Economic theory considers that employment protection legislation has different effects depending on whether it specifies a severance payment to be paid to the worker in case of dismissal or it imposes a tax on the firm in the form of legal procedures, etc. In the first case this is a transfer internal to the firm/worker match which in many bargaining models will be offset and does not affect hiring nor separation decisions. In the second case the match is transferring resources to the rest of the world upon separation and this will typically affect hiring and separations decision. See Lazear (1990) and Burda (1992). Empirically, in European countries both the severance payment component and the tax component are large.
} 
forces the economy to pay the firing cost at least part of the time, thus ensuring that utility eventually falls as firing costs increase. Intuitively, it can be given several interpretations. For example this can be interpreted as the worker quitting (because of exogenous geographical mobility, say), and the administration misinterpreting the move and imposing the firing tax upon the firm a fraction $\rho$ of the time. Or it could be a large adverse shock forcing the plant to close, with the administration, again, imposing the tax over a fraction $\rho$ of such events.

Firms and workers can freely borrow and lend at the real interest rate $r$, which is exogenous. We assume $r>g$ which guarantees that the present discounted value of income streams will be well defined.

Wage formation is not competitive: wages are set above market clearing level, in a way specified below. In equilibrium there is a positive stock of involuntarily unemployed workers who wait for finding a job with a new entrant. Firms, by contrast, can fill vacancies instantaneously. ${ }^{4}$

Below the variable $s$ will refer to the date at which a match is established, while $t$ refers to the current date. Let $J(s, t)$ be the value as of $t$ of a firm that entered the market at $s$. Let $w(s, t)$ be the wage in that firm. Then the evolution equation of $J$ is

$$
r J(s, t)=a_{s}-w(s, t)-\delta\left(\rho F a_{t}+J(s, t)\right)+\frac{\partial}{\partial t} J(s, t)
$$

The free entry condition implies that

$$
J(s, s)=0
$$

The firm/worker match will leave the market at the date $T(s)$ when its

\footnotetext{
${ }^{4}$ Thus, the model is different from a matching model where both sides of the market have to wait. Here there is no "friction", just a wage that does not clear the market, so that only one side is rationed. The logic is the same as in Shapiro and Stiglitz (1984).
} 
value is just equal to the negative of the firing cost: ${ }^{5}$

$$
J(s, T(s))=-F a_{T(s)}
$$

(1) and (3) imply that

$$
J(s, t)=\int_{t}^{T(s)}\left(a_{s}-w(s, u)-\delta \rho F a_{u}\right) e^{-(r+\delta)(u-t)} d u-F a_{T(s)} e^{-(r+\delta)(T(s)-t)}
$$

The firm sets its exit time optimally by maximizing (4) with respect to $T(s)$. The first order condition is:

$$
w(s, T(s))-a_{s}=(r+\delta(1-\rho)-g) F a_{T(s)}
$$

The LHS is the loss per period made by the firm while the right-hand side is the annuity value of the firing cost. If the firing cost is large enough it may be optimal for the firm to never fire the worker but let him go when hit by the shock $\delta$. In that case the condition is:

$$
w(s, t)-a_{s}<(r+\delta(1-\rho)-g) F a_{t}, \forall t \geq s
$$

We now turn to wage determination. We make the simple assumption that workers can appropriate a share of the surplus generated by the match

\footnotetext{
${ }^{5}$ In equilibrium the value of the firm is always negative except when it enters the market. Profits are positive at the beginning, but negative at the end. This feature, however, would disappear if we were to allow for a strictly positive entry cost. In that case the value of the firm will be strictly positive for a time interval of strictly positive measure after it has entered the market, but would eventually become negative for a while until the position is closed.

Note that we assume that shareholders are in some way compelled to put up the money needed to cover the losses in the latter stage of the firm's life cycle. One possibility is to assume that they are making provisions during the phase with positive profits, another that they belong to conglomerates holding representative portfolios of the economy's productive structure. These conglomerates cannot sell their negatively valued units nor close them without paying the firing costs. The legislation is thus constraining them to cross-subsidize the least efficient units.
} 
gross of the firing cost. That is, we assume that at any point in time wages are set so that:

$$
V_{e}(s, t)=V_{u}(t)+\varphi S(s, t)
$$

, where $V_{e}$ is the present discounted utility of an employed worker, $V_{u}$ that of an unemployed worker, and $S(s, t)$ is the present discounted value of the match's output:

$$
S(s, t)=\int_{t}^{T(s)} a_{s} e^{-(r+\delta)(u-t)} d u
$$

This formulation is simple and has two advantages. First, firing costs do not affect the wage formation process, allowing us to insulate their pure employment protection effects, while their bargaining power is captured by the parameter $\varphi$. Second, because they are able to extract part of the gross surplus, workers will be willing to continue to work for the firm even though it is making losses. ${ }^{6}$ (7) is slightly different from what ordinary bargaining models implied, but can be given microfoundations as a version of the efficiency wage model (see appendix).

The evolution equations for $V_{e}$ is:

$$
r V_{e}(s, t)=w(s, t)+\frac{\partial V_{e}}{\partial t}(s, t)+\delta\left(V_{u}(t)-V_{e}(s, t)\right)
$$

\footnotetext{
${ }^{6}$ Even though firing costs cannot be bargained out between the firm and the worker because they is paid to a third party, they turn out to be inoperative under ordinary Nash bargaining if workers are given the option to voluntarily leave the firm, as is reasonable; This is because if $J<0$ then one must have $V_{e}<V_{u}$. But then the worker would prefer to quit. So as long as quitting is not taxed, the firm's net value cannot be negative and inefficient matches cannot continue.

One solution to this problem is to assume that $F$ must be paid by the firm upon disagreement. Nash bargaining then amounts to maximizing $\left(V_{e}-V_{u}\right)^{\psi}\left(J+F a_{t}\right)^{1-\psi}$. In this formulation, the firm's threat point is $-F a_{t}$ rather than 0 . The problem with that is that $F$ then plays the twin role of preventing inefficient matches from closing and enhancing the worker's bargaining power. This is not unreasonable, but from an analytical point of view it is much better to have a model where employment protection and bargaining power are two captured by two independent parameters. This is what our assumption on wage formation buys us.
} 
Note that on the job search is ruled out: any separation must result in an unemployment spell. The terminal condition for $V_{e}$ is $V_{e}(s, T(s))=V_{u}(T(s))$, which will always hold because of (7) and (8).

We assume that the unemployed earn an income flow equal to $b a_{t} . b$ can be positive or negative. A negative $b$ means that there are important search costs and disutilities associated with being unemployed, that outweigh unemployment benefits. The main role of $b$ is not solely to capture unemployment benefits but rather to add one degree of freedom that allows to pin down the unemployment level by controlling the intensity with which the unemployed compete for jobs. The lower $b$, the more eager the unemployed are to get jobs and the lower the unemployment rate. ${ }^{7}$

Therefore, $V_{u}$ evolves according to:

$$
r V_{u}(t)=b a_{t}+\frac{d V_{u}}{d t}(t)+\theta_{t}\left[V_{e}(t, t)-V_{u}(t)\right]
$$

$\theta_{t}$ is the equilibrium probability per unit of time of finding a job; it is typically finite because insider bargaining power creates involuntary unemployment. The term in brackets is the capital gain made when a job is found. The value of the next job to the worker is $V_{e}(t, t)$, since he will hold a newly created job.

\section{Solution of the model}

We are now in a position to solve the model for a constant exogenous level of the firing cost. The key variables of interests are $T(t)$, the duration of a match, and $\theta_{t}$, the level of labor market tightness.

First, one can get an expression for wages by eliminating $V_{e}(s, t)$ and

\footnotetext{
${ }^{7}$ A negative $b$ would be equivalent to adding $b a_{t}$ to the wage of the employed instead, that is, in addition to their bargained wage the employed get some "piece rate" payment on a machine which is constantly updated to the latest technology.
} 
$V_{u}(t)$ between $(7),(9)$, and $(10)$, and using (8) to compute $\partial S / \partial t$ :

$$
w(s, t)=b a_{t}+\theta_{t} \varphi S(t, t)+\varphi a_{s}
$$

This wage equation is similar to what is found in many models. The wage is the sum of three terms. The first term is unemployment benefits; the second term is the contribution to the outside option of the expected surplus an unemployed worker will extract in his next job. The third term is the flow of surplus extracted from the current job. Note that firing costs do not directly affect the wage formation equation.

Substituting (11) into (4) and making use of the free entry condition (2) we get a first relationship between $\theta$ and $T(s)$ :

$$
\begin{aligned}
0= & (1-\varphi) S(t, t)-\varphi \int_{t}^{T(t)} \theta_{u} S(u, u) e^{-(r+\delta)(u-t)} d u \\
& -b \int_{t}^{T(t)} a_{u} e^{-(r+\delta)(u-t)} d u \\
& -\int_{t}^{T(t)} \delta \rho F a_{u} e^{-(r+\delta)(u-t)} d u-F a_{T(t)} e^{-(r+\delta)(T(t)-t)}
\end{aligned}
$$

, where $S(.,$.$) is defined by (8)$.

(12) defines a relationship between $T(t)$, the path of stopping dates, and $\theta_{t}$, the path for labor market tightness that must hold for the free entry condition to be satisfied. Note however that we have ignored the corner case $\theta=0$, in which case profits have to be negative rather than zero and the $=$ sign must be replaced by $\mathrm{a} \geq$.

Substituting (11) into the first-order condition (5) we get the second relationship between $\theta$ and $T(s)$ :

$$
(\varphi-1) a_{t}+b a_{T(t)}+\theta_{T(t)} \varphi S(T(t), T(t))=(r+\delta(1-\rho)-g) F a_{T(t)}
$$

This is the relationship that must hold for stopping dates to be optimal, although we are again ignoring the "corner" case $T(t)=+\infty .^{8}$

\footnotetext{
${ }^{8}$ In this regime, the LHS of (13) must be $\leq$ to the RHS.
} 
A solution to the model is a path for $\left(\theta_{t}, T(t)\right)$ that satisfies both (12) and (13) for any $t \geq 0$. The implied path for the other variables of interest, $w(s, t), V_{e}(s, t)$, and $V_{u}(s, t)$ can then be computed using (9)-(11).

\subsection{Existence}

A natural solution to look for is a balanced growth path, namely a solution to (9)-(13) where $V_{u}$ grows at a constant rate, $\theta$ and $T(t)-t$ (the obsolescence age of a job) are constant, and $V_{e}$ and $w$ computed at a given plant age grow at a constant rate ${ }^{9} .{ }^{10}$ It should be noted that neither the initial level of unemployment nor the initial distribution of employment across firms enter in (9)-(13). Therefore if a balanced growth path is solution then the values of $\theta, T, w, V_{e}, V_{u}$ can jump to that solution regardless of initial conditions. This property will be very convenient when it comes to analyzing the impact of reform.

The following proposition shows that such a balanced path exists:

\section{PROPOSITION 1 -}

Assume $F>0$ and

$$
b(r+\delta) /(1-\varphi)<r+\delta(1-\rho)-g
$$

1. For any given value of $F$ constant from $t=0$ on there exists an equilibrium such that

\footnotetext{
9i.e., $\partial w(t-z, t) / \partial t=g . w(t-z, t)$, $\partial V_{e}(t-z, t) / \partial t=g \cdot V_{e}(t-z, t)$.
}

${ }^{10}(13)$ implies that in an equilibrium where $\theta$ is constant, the second order condition of the optimal stopping condition is always satisfied. To see this, note that taking the second derivative of (4) with respect to $T(s)$, we get a second order condition which is

$$
\partial w(s, T(s)) / \partial t \geq(r+\delta(1-\rho)-g) F g a_{T(s)}
$$

The wage equation (11) yields $\partial w / \partial t=b g a_{t}+g \theta \varphi S(t, t)$. (13) implies that this is equal to $(r+\delta(1-\rho)-g) F g a_{t}+(1-\varphi) g a_{t} \geq(r+\delta(1-\rho)-g) F g a_{t}$, so that the SOC is always satisfied. 
(i) $\theta_{t}=$ constant and $T(s)=s+x, \forall s \geq 0$ with $x=$ constant $\in[0,+\infty]$

(ii) $V_{e}(s, t)$ grows at a constant rate $g$ with respect to $s: \partial V_{e} / \partial s=g V_{e}$, $V_{u}$ grows at constant rate $g$.

2. If $F<(1-\varphi) /(r+\delta), x$ is finite and $\theta$ is strictly positive. They are solution to the following system of equations:

$$
\begin{gathered}
(1-\varphi)\left[\frac{1}{r+\delta}+\frac{g e^{-(r+\delta) x}-(r+\delta) e^{-g x}}{(r+\delta)(r+\delta-g)}\right]=F \\
{[\theta \varphi+g(1-\varphi)] \frac{1-e^{-(r+\delta) x}}{r+\delta}=1-(b+\delta \rho F+\varphi)}
\end{gathered}
$$

3. If $(1-\varphi) /(r+\delta) \leq F<\frac{1}{\delta \rho}\left[(1-\varphi)\left(\frac{r+\delta-g}{r+\delta}\right)-b\right]$, then $x$ is infinite and $\theta>0$. $\theta$ is solution to (16) with $x=+\infty$, i.e.

$$
\theta \varphi+g(1-\varphi)=1-(b+\delta \rho F+\varphi)
$$

4. If $F \geq \frac{1}{\delta \rho}\left[(1-\varphi)\left(\frac{r+\delta-g}{r+\delta}\right)-b\right]$, then $x=+\infty$ and $\theta=0$.

PROOF-See Appendix

The essence of proposition 1 is contained in statements 1 and 2, which tell us that an equilibrium exists and how to compute it. (15) uniquely determines $x$ as a function of the parameters, since the LHS is increasing in $x$. There exists a solution for any $F<(1-\varphi) /(r+\delta)$. As $F$ converges to that value, the implied obsolescence age $x$ converges to infinity. Once $x$ is computed, (16) allows to compute $\theta$, the equilibrium level of market tightness.

Statements 3 and 4 take care of special cases that turn out to be irrelevant. Assumption (14) guarantees that even for a firing cost that prevents firms from ever becoming obsolete, i.e. $F=(1-\varphi) /(r+\delta)$, unemployment does 
not jump to $100 \%$-i.e., labor costs are not large enough to deter entry. As firing costs rise beyond that level, $x$ remains infinite, and the economy eventually reaches a zone where everybody is unemployed. However, given that $F=(1-\varphi) /(r+\delta)$ achieves the maximum possible level of employment protection, nobody would want to increase $F$ beyond that point.

Therefore, in the remainder of the paper, we shall assume that (14) holds and allow $F$ to vary between 0 and $(1-\varphi) /(r+\delta)$, meaning that $x$, the duration of a plant, varies between 0 and $+\infty$. Therefore, equilibrium will always be characterized by (15)-(16), or their limits.

\subsection{Properties}

We now discuss the properties of the equilibrium, starting with how parameters affect $x$.

PROPOSITION 2 - Assume (14) and $F<(1-\varphi) /(r+\delta)$. Then the impact of parameters on $x$ is summarized by the following: :

$$
x=x\left(\begin{array}{llll}
r+\delta & g & F & \varphi \\
+ & , & + \\
+
\end{array}\right)
$$

PROOF - See Appendix.

The economic interpretation behind proposition 2 is as follows: growth in the rest of the economy drives wages up as time passes in any given match. At some point they exceed output, so that the firm wants to get rid of the worker (economic obsolescence). Firing costs create an incentive to delay that moment because it is optimal to pay the firing cost only if costs are sufficiently above revenues. Thus $x$ increases with $F$. A faster growth rate increases the pace of obsolescence via more rapid wage growth within existing matches. Consequently $x$ falls with $g$. A higher interest rate makes it more 
profitable to postpone expenditure on the firing cost, and a higher exogenous rate of separation acts in the same direction. Finally, a higher bargaining power for workers $\varphi$ also increases the duration of matches. That comes from the first term in (29) and the fact that firing costs also act as hiring $\operatorname{costs}^{11}$; here the cost of setting up a match consists of the expected associated firing costs; in equilibrium cumulated profits must be large enough to pay for that cost; when $\varphi$ increases the firm's profit flow is lower, so that it must stay longer in business to cover the firing cost.

We now turn to the comparative statics with respect to $\theta$.

PROPOSITION 3 - In equilibrium $\theta$ can be expressed as a function of $x$ and of $b, \varphi, g, r$ :

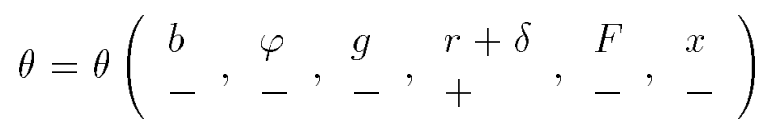

Consequently, the reduced form impact of parameters on $\theta$ is given by

$$
\theta=\theta\left(\begin{array}{lllll}
b & \varphi & g & r+\delta & F \\
- & - & ?
\end{array}\right)
$$

PROOF - Straightforward from inversion of (16) $\cdot{ }^{12} \square$

The impact of $b, \varphi$, and $F$ is clear: all these parameters increase labor costs and reduce the firm's value and the incentive to enter the market. To restore these incentives wages must go down which can only happen with more slack in the labor market. The direct negative effect of $g$ is due to the fact that faster growth increases the growth rate of wages of any given job and consequently, as that job's productivity does not grow, reduces incentives to enter the market. On the other hand, faster growth reduces the length

\footnotetext{
${ }^{11}$ A point made by Bertola (1990).

${ }^{12}$ Note that (16) also holds in the limit case where $F \geq(1-\varphi) /(r+\delta)$ and $x=+\infty$.
} 
of matches, which reduces the surplus appropriable by the worker, which in turn depresses wages, thus boosting entry and labor market tightness. The net, reduced form effect of $g$ is therefore ambiguous.

Next, it is possible to characterize the equilibrium unemployment rate and the steady-state distribution of employment across vintages. Aggregate employment in firms of age $z$ decays at rate $\delta$, and $z$ is between 0 and $x$, the obsolescence age. Therefore in steady state the density of employment in firms aged $z$ is ${ }^{13}$

$$
g(z)=\frac{\delta e^{-\delta z}}{1-e^{-\delta x}}, 0 \leq z \leq x
$$

Let now $l=1-u$ be the employment rate. The outflow from unemployment is $\theta(1-l)$, while the inflow is $l(\delta+g(x))$. The first term comes from the exogenous separation source, the second from the inflow of matches reaching the obsolescence age $x$. This allows to compute the steady-state unemployment rate as a function of $\theta$ and $x$ :

$$
u=\frac{\delta}{\delta+\theta\left(1-e^{-\delta x}\right)}
$$

It should be noted that unemployment is not necessarily higher when firing costs are lower. A lower firing cost increases $\theta$, which tends to reduce $u$, but reduces $x$, which tends to increase $u$. Job creation is higher but so is job destruction, so unemployment may rise or decline. This is now well known from the analysis of firing costs. ${ }^{14}$ On the other hand, firing costs unambiguously increase unemployment duration, which on average is $1 / \theta$.

One should also point out that the model captures the "creative destruction" effects of growth on unemployment discussed by Aghion and Howitt (1994). We have seen that faster growth has ambiguous effects on labour market tightness, and also that $x$ falls when $g$ increases. This latter effect means

\footnotetext{
${ }^{13}$ This distribution has by convention a total mass equal to one, not the employment rate.

${ }^{14}$ Bentolila and Bertola (1990); Bentolila and Saint-Paul (1994).
} 
that growth increases the pace of obsolescence and therefore job destruction, which, in accordance to (19), tends to increase unemployment. Whereas Aghion and Howitt postulated that a faster pace of innovation increased job destruction because of the retraining needs, here faster growth increases job destruction endogenously because of its effect on the obsolescence decision.

One can further compute aggregate output in steady state, as it is equal to $Y_{t}=y a_{t}=(1-u) a_{t} \int g(z) e^{-g z} d z$, implying

$$
y=\frac{\delta \theta}{\delta+\theta\left(1-e^{-\delta x}\right)} \frac{1-e^{-(g+\delta) x}}{g+\delta}
$$

\subsection{The $\mathrm{F}=0$ case}

It is worth discussing the limit case where the firing cost is zero. If $F=0$ then (15) implies $x=0$ : matches only last an infinitesimal amount of time ${ }^{15}$. Substituting $x=0$ into (16) implies that $\theta$ must be infinite, that is, people find jobs instantaneously. Finally the wage formation equation implies $V_{e}=$ $V_{u}$.

These properties also obtain in the Walrasian allocation where the whole workforce uses the state of the art technology at any point in time, so that all existing matches are constantly destroyed and there workers constantly reallocated to new matches.

Does, then, the equilibrium with $F=0$ match the first best? The answer is in general no, except when $\varphi=0$. Workers alternate infinitesimal spells of employment with infinitesimal spells of unemployment in such a way that the fraction of time spent in unemployment is strictly positive unless $\varphi=0$. (19) and (16) imply that as $x$ goes to $0, u$ converges to $\varphi /(1-b){ }^{16}$

\footnotetext{
${ }^{15}$ This would not be true if there was an entry cost.

${ }^{16}$ Consider (19). When $x$ goes to zero it is equivalent to $1 /(1+\theta x)$. (16) then implies that at zero firing costs $\theta x=(1-(b+\varphi)) / \varphi$. Thus the unemployment rate at zero firing cost is given by $u=\varphi /(1-b)$.
} 
Finally wages can be computed in that equilibrium; it can be checked that $w_{t}=a_{t}$, the maximum possible wage. That is not surprising, as all existing jobs have a productivity $a_{t}$ at any point in time, and free entry would eliminate any discrepancy between $w_{t}$ and $a_{t} \cdot{ }^{17}$

With that discussion we conclude the characterization of equilibrium. We now proceed and discuss voting on firing costs.

\section{Voting on firing costs}

From $t=-\infty$ to $t=0$ the economy has been in a steady state corresponding to $x_{0}$ and $F_{0}$, respectively. At $t=0$ people vote once and for all on a firing cost to prevail from now on.

We will say that $F$ is a political equilibrium if it defeats any other alternative.

$F$ is a local political equilibrium if there exists $\varepsilon>0$ such that $F$ defeats any alternative in the $[F-\varepsilon, F+\varepsilon]$ interval.

$F$ is a stationary political equilibrium (resp. stationary local political equilibrium) if it is a political equilibrium (resp. local political equilibrium) for $F_{0}=F$.

We first discuss the preferences for employment protection, which will allow us to sort out the winners and losers from employment protection regulation.

\footnotetext{
${ }^{17}$ Algebraically we have that $w_{t} / a_{t}=b+\varphi e^{-g z}+\theta \varphi\left(1-e^{-(r+\delta) x}\right) /(r+\delta)$. At $x=0$ this is equivalent to $w_{t} / a_{t}=b+\varphi+\varphi \theta x$, which converges to 1 .
} 


\subsection{The shape of preferences for employment protec- tion}

For simplicity we assume that shareholders represent a negligible share of the population, at least in terms of political power. ${ }^{18}$ People's preferences are therefore determined by the utility associated with their labor market status, as determined in the previous section. At the time of voting, workers differ according to whether they are employed or unemployed, and employed workers differ according to how long they have been in their job. Thus, job duration $z$ is initially distributed over $\left[0, x_{0}\right]$ with a density given by (18). We also assume, realistically, that the initial level of unemployment is less than 0.5 , so that the decisive voter will not be unemployed.

The first step is to compute the utility of the employed and unemployed voters as a function of the collectively decided firing cost. As stated above, regardless of the initial conditions the value functions jump to their steady state level at the time $F$ is permanently changed. That is, the system of equations which determines $w, \theta, x, V_{e}$ and $V_{u}$ is independent of the distribution of people between employment and unemployment and across plant ages. At the time of a change in $F$ all these variables jump to their new steady-state values as determined in the previous section. If $x<x_{0}$ then those plants initially older than the new optimal value of $x$ find themselves beyond the obsolescence margin and instantaneously close at the time of reform.

Therefore, it is correct to use the above formulas to compute the gains

\footnotetext{
${ }^{18}$ When firing costs increase, existing firms make capital losses, by virtue of the envelope theorem. Capitalists are therefore always in favour of the lowest possible value of the firing cost. Assuming a fixed number of capitalists who vote would therefore only shift the decisive voter's position by that number.

Things would obviously be more complicated if workers owned shares. The greater the capital they own, the lower their marginal gain from increasing firing costs. Preferences for labor market rigidity then depends on the relative importance of labor vs. capital endowment. This logic applies to any institution that redistributes from firms to workers, or between two factors of production, including minimum wages, tariffs, etc. Here, however, we are chiefly concerned with conflicts of interests among workers.
} 
and losses from reform.

Using (7) and (10) along the balanced growth path (where $V_{u}$ grows at rate $g$ ) we can compute the utility of the unemployed:

$$
V_{u}(t)=\frac{a_{0} e^{g t}}{r-g}\left[b+\theta \varphi \frac{1-e^{-(r+\delta) x}}{r+\delta}\right]
$$

, which, given (16), is equivalent to

$$
V_{u}(t)=\frac{a_{0} e^{g t}}{r-g}\left[(1-\varphi)\left(1-g \frac{1-e^{-(r+\delta) x}}{r+\delta}\right)-\delta \rho F\right]
$$

By making use of (7) again we can recover the utility of an employed worker in a firm of age $z$ :

$V_{e}(z, t)=a_{0} e^{g t}\left[-\frac{\delta \rho F}{r-g}+\left(1-g \frac{1-e^{-(r+\delta) x}}{r+\delta}\right) \frac{1-\varphi}{r-g}+\varphi e^{-g z} \frac{1-e^{-(r+\delta)(x-z)}}{r+\delta}\right]$

The last term in the brackets represents the present discounted value of the rent to be earned until the current job elapses. It is larger, the larger the voted value of $x$, and smaller, the larger the current age of the job $z$. The term in $-\delta \rho F$ in (20) and (21) is the direct burden of firing costs, due to the fact that part of them has to be paid upon exogenous separation and that this cannot be offset by reducing the separation rate as is the case with the obsolescence decision.

In (20) and (21) $F$ is implicitly a function of $x$ as defined by (15). Thus voting on $F$ is equivalent to voting on $x$. For analytical purposes it turns out to be simpler to use $x$ rather than $F$ as the policy variable, which we do in the sequel. When $x$ spans $\mathrm{R}^{+}, F$ spans $\left[0, \frac{1-\varphi}{r+\delta}\right]$. This is the relevant interval of values of $F$ over which people vote. ${ }^{19}$

\footnotetext{
${ }^{19}$ At this stage we can check that it is never optimal to vote for $F>(1-\varphi) /(r+\delta)$. In that zone $x$ should be replaced with $+\infty$ in (20) and (21), and the only term that varies with $F$ is $-\delta \rho F$, which is decreasing in $F$.
} 
It is important to note that (21) is only valid of $x \geq z$. Once people have voted on $x$ all firms with age $z>x$, if any, instantaneously disappear and fire their workers. The utility of an employed when $z>x$ is thus given by $V_{u}$.

The following lemmas tells us how the preferences of an employed in a firm of age $z$ vary with $x$.

LEMMA 1 - The RHS of (20) is monotonically decreasing in $x$.

PROOF- Straightforward differentiation.

LEMMA 1 tells us that the unemployed prefer the lowest possible value of $x$ (or, alternatively, $F$ ). In the $F=0$ equilibrium people move constantly between employment and unemployment so that it is as if the total amount of work were perfectly shared among people. The incumbent employee's advantage for tomorrow's jobs is eliminated; as this equilibrium yields the highest probability of finding a job and the highest wage, it is the one preferred by the unemployed.

LEMMA 2- The RHS of (D1) is

(i) decreasing in $z$

(ii) decreasing in $x$ if and only if $\varphi e^{(r+\delta-g) z} \leq(1-\varphi) g /(r-g)$

(iii) increasing and then decreasing in $x$ if and only if $\varphi e^{(r+\delta-g) z}>(1-$ $\varphi) g /(r-g)$.

(iv) At any given $x$ the derivative of the RHS of (21) with respect to $x$ is larger, the larger $z$.

PROOF- See Appendix 
This lemma implies that for any given $x$ workers in older firms have a lower utility than workers in younger firms, both because of lower productivity and shorter remaining employment duration. It also tells us, roughly, that people like employment protection more, the smaller $g$, the larger $r$, the larger $\varphi$, and the larger $z$. This has important consequences for the characteristics of the equilibrium, which we discuss below.

Figure 1 illustrates how preferences depend on $x$ for various types of workers. The downward sloping curve $C_{u}$ represents the preferences of an unemployed worker. $C_{0}$ represents the preferences of a worker at a newly created plant. $C_{z}$ represents the utility of a worker at a plant of age $z$ for $x>z$. For $x<z$ his utility is given by $C_{u}$. $C_{z^{\prime}}$ represents the utility of a worker with $z^{\prime}>z$. As $z$ increases, $C_{z}$ shifts down and its peak shifts further to the right. ${ }^{20}$

\subsection{Condorcet cycles and the "lost generation effect"}

As Figure 1 makes clear, preferences are not single-peaked (nor single-crossed), so that one cannot readily apply a median voter theorem to compute the political equilibrium. A potential for Condorcet cycles and non-existence exists. The underlying logic is illustrated in Figure 2.

In figure $2, x^{*}$ is a candidate stationary equilibrium. It is the preferred point of some decisive voter $z_{m}$.

In figure $2 \mathrm{a}$ we consider a contest between $x^{*}$ and a small increase in firing costs to $x^{+}$. Then, a consequence of lemma 2 is that (i) there is a worker $z^{+}$ $\geq z_{m}$ who is indifferent between $x^{*}$ and $x^{+}$; (ii) all workers such that $z \geq z^{+}$ prefer $x^{+}$(Group 2); and (iii) all workers such that $z \leq z^{+}$prefer $x^{*}$ (Group 1). Thus it seems that things work out as in the median voter theorem, with

\footnotetext{
${ }^{20}$ In drawing figure 1 we have assumed $\varphi>(1-\varphi) g /(r-g)$. That condition implies $\varphi /(1-\varphi)>\rho \delta g /(r+\delta-g) /(r-g)$, which, according to equation (42) in the appendix, implies that the RHS of (21) is upward sloping at $x=z$.
} 
people in older plants preferring higher firing costs.

That conclusion does not hold, however, when we deal with reductions in firing costs from $x^{*}$ to $x^{-}$.

This is because those who are about to lose their jobs at $x=x^{*}$ are in the worst possible situation. Given that they will be unemployed very soon they prefer a more flexible society that increases their chances of finding a job, although they would also benefit from an increase in rigidity that would maintain them into their jobs for longer.

Thus, as figure $2 \mathrm{~b}$ makes clear, there are now two indifferent workers. One is given by $z^{-} \leq z_{m}$, the worker who remains employed under $x^{-}$and is indifferent between $x^{*}$ and $x^{-}$. The other is given by $\hat{z} \in\left[x^{-}, x^{*}\right]$, the worker who is indifferent between being employed (for a short time) in the rigid society and being unemployed in the flexible one. $x^{-}$is preferred to $x^{*}$ by all workers such that $z<z^{-}$(Group 1 ), but also by all those such that $\hat{z} \leq z \leq x^{*}$. (Group 3) Therefore, it is no longer true that rigidity is preferred by workers at older plants. Workers at very old plants prefer the flexible society over the rigid one. They will soon be constrained to a "new start" anyway, and the flexible society is the one that gives them the best chances.

It is the existence of this "lost generation" that provides the potential for Condorcet cycles. Figure 3 illustrates that possibility, when the three alternatives considered are $x^{*}$ (the status quo), $x^{+}$, and $x^{-} \cdot z_{m}, z^{+}, z^{-}$, and $\hat{z}$ are defined as above. $\tilde{z}^{e}$ denotes the employed worker who is indifferent between $x^{-}$and $x^{+} . z^{u}$ denotes the worker who is indifferent between $x^{-}$and $x^{+}$and loses his job under $x^{-21}$.

Let the number of employed people in the intervals $\left[0, z^{-}\right],\left[z^{-}, \tilde{z}^{e}\right],\left[\tilde{z}^{e}, z^{+}\right]$, $\left[z^{+}, \hat{z}\right],\left[\hat{z}, \tilde{z}_{u}\right]$, and $\left[\tilde{z}_{u}, x^{*}\right]$ be denoted by $A, B, C, D, E, F$ respectively and let

\footnotetext{
${ }^{21}$ One clearly has $\tilde{z}^{e}>z^{-}$, since $x^{+}$is more rigid that $x^{*}$ and since $z^{-}$. Also $\tilde{z}^{u}>\hat{z}$, since $x^{+}$makes a worker with $z=\hat{z}$ better-off relative to $x^{*}$.
} 
$u$ the number of unemployed people. Then clearly there are $u+A+E+F$ people favoring $x^{-}$over $x^{*}, u+A+B+C$ people favoring $x^{*}$ over $x^{+}$, and $C+D+E$ people favoring $x^{+}$over $x^{-}$. It may well be that these 3 quantities are all greater than $1 / 2$, in which case there will be a Condorcet cycle. The lost generation is willing to make a coalition with either those who support lower firing costs (relative to $x^{*}$ ) or those who support higher firing costs, thus generating instability in the voting process.

\subsection{Equilibria}

Despite the Condorcet cycle problem, we are able to establish a number of results concerning local political equilibria, and in some cases global equilibria as well.

The following proposition tells us when full flexibility $(F=x=0)$ can be an equilibrium.

\section{PROPOSITION 4 -}

(i) Assume

$$
\varphi \leq g / r
$$

, then for all initial distributions of $z$ and for all values of $F_{R}$, the unique political equilibrium is $F=0$.

(ii) Furthermore, (2D) is necessary and sufficient for $F=0$ to be a stationary local political equilibrium.

PROOF - see appendix.

Figure 4 illustrates the proof of proposition 4 . If (22) holds then the preferences of a worker in a new plant are decreasing with $x$. Thus, these 
people are worse-off at $x>0$ than an unemployed at $x=0$. But, given that they are better-off at any $x$ than those in older plants, it must follow that all the employed have their maximum utility at $x=0$.

Proposition 4 implies that for a "flexible" society to be stable (in the sense that people will not want to change its institutions), it must be the case that the worker's share of the surplus does not exceed the ratio between the growth rate and the interest rate.

Can we prove that when $\varphi \geq g / r$, there exists a political equilibrium with a strictly positive value of $F$ ? This is almost true, but because of the Condorcet problem we must content ourselves with a more limited result.

PROPOSITION 5 - Assume

$$
\varphi \geq g / r
$$

and

$$
\frac{(r+\delta(1-\rho)-g)^{2}}{\delta \rho(r+\delta-g)} \leq \frac{\varphi(r-g)}{g(1-\varphi)}
$$

Then

(i) there exists a stationary local political equilibrium such that $F>0$.

(ii) in such an equilibrium the collectively chosen value of $F$ (or equivalently $x)$ maximizes $V_{e}\left(x, z_{m}\right)$ where $z_{m}$ represents the plant age of the median voter, i.e. it satisfies

$$
(1-u) \int_{z_{m}}^{x} \frac{\delta e^{-\delta z}}{1-e^{-\delta x}} d z=1 / 2
$$

, where $u$ is the steady state unemployment rate corresponding to $x$.

PROOF -See Appendix.

Condition (23) is a sufficient condition that guarantees that when we run a contest between the status quo and a marginal reduction in firing costs, the 
size of the "lost generation" is not large enough to overturn the status quo. It will be satisfied if $g$ is small enough, $r$ large enough, or $\varphi$ large enough, i.e. if one violates (22) by a sufficient margin. (23) can also be satisfied, for any given $(r, \varphi, g)$, for $\rho$ close to 1 and at the same time $\delta$ sufficiently large.

Figure 5 illustrates proposition 5 . The horizontal axis represents $x$, the equilibrium value of plant duration. The vertical axis represents the plant age of the decisive voter. The upward sloping VV curve plots, as a function of $x$, the plant age of an employed agent whose bliss point is $x$. That is, it tells us that the equilibrium value of $x$ must be the preferred point of the decisive voter and gives us the plant age of that decisive voter. $V V$ is below the horizontal axis for $x$ small enough, meaning that if (22) is violated workers with $z=0$ have a strictly positive most preferred value of $x$. As $x$ gets larger, it is the most preferred value of workers at older plants. One can show that VV goes to infinity as $x$ goes to infinity, with an asymptote parallel to the 45-degree line (see appendix). ${ }^{22}$

The SS curve represents the plant age of the median voter, $z_{m}$, conditional on the economy being initially in a steady state corresponding to $x$. It is determined by the condition that there are as many employed workers in plants older than $z_{m}$ as either unemployed or working at younger plants, i.e. (24) or equivalently:

$$
\frac{\theta}{\delta+\theta\left(1-e^{-\delta x}\right)}\left[e^{-\delta z_{m}}-e^{-\delta x}\right]=1 / 2
$$

where $u$ and $\theta$ are the steady-state values corresponding to $x$.

$z_{m}$ is typically increasing with $x$, since a larger $x$ shifts the initial distribution of employment toward older plants, although not necessarily, since a larger $x$ may also increase unemployment, and the unemployed are counted on the left of the median.

\footnotetext{
${ }^{22}$ In terms of the underlying policy variable $F$, when $x$ goes to infinity $F$ converges to $(1-\varphi) /(r+\delta)$.
} 
For $x=0$ one clearly has $z_{m}=0$ since there are only newborn plants in the economy. As $x$ goes to infinity $z_{m}$ converges toward a finite value $\bar{z}$ which is computed using (25) and (17). Thus, as figure 6 makes clear, SS is above VV for low values of $x$ and below it for large values of $x$. Consequently there exists a point where they cross, and where VV is locally steeper than SS. This point is a stationary local political equilibrium. The induced distribution of employment is such that the bliss point of the median voter is precisely the status-quo.

\subsection{Discussion}

The two preceding propositions summarize the main message of the paper: firing costs are likely to emerge in economies with high rents to incumbent workers ( $\varphi$ large), a low rate of creative destruction ( $g$ small) and a high rate of time preference ( $r$ large).

When evaluating the gains from an increase in firing costs, workers trade off an increase in the length of time over which they earn their rent against a reduction in living standards due both to lower average productivity and a larger direct burden of the legislation.

The gains from employment protection depend on employee rents. At $\varphi=0$ there is no utility loss associated with losing one's job. In such a case nobody would support firing costs: the benefits from increasing tenure are zero. A higher worker's share increases the support for employment protection: the value of increasing tenure is larger, the larger the employed's rent.

The employed are more likely to benefit from an increase in firing costs when the interest rate is higher because that increases the weight of the current job's longer duration in the employed's utility, relative to the future lower utility when the worker is unemployed.

The growth rate acts in two ways. First it increases the obsolescence rate 
and therefore the cost of maintaining unproductive matches idle rather than reallocating their workers to the most up-to-date technology. (This cost in equilibrium is passed to worker in the form of lower wages). This in itself reduces the support for employment protection. Second, faster growth tends to reduce the effective discount rate applied to the future: incumbent workers put more weight on the lower job finding rate they will experience once their current match is dissolved, because future jobs pay more. This also tends to reduce the support for employment protection.

Finally, workers at older plants have a greater marginal gain from increasing firing costs because they discount the event of job loss at a lower rate than workers at young plants. Therefore, an increase in firing costs is supported by workers at plants older than the one of the decisive voter. But, as we have already discussed, things are more complicated when considering a reduction in firing costs, because of the lost generation effect.

In principle, VV and SS may cross more than once, as they typically have the same slope. One may then have multiple steady states. This is due to the fact that worker at older plants prefer higher firing costs (provided they keep their jobs), so that an increase in the age distribution of firms is selfreinforcing through the political system. However, the numerical simulations below suggest that VV is always far steeper than SS, so that multiple equilibria are ruled out. What remains, though, is a multiplier effect:an increase in preferences for employment protection (i.e., a rightward shift of VV) is further reinforced by the increase in the median voter's plant age that it induces.

The next section establishes some local comparative statics result for the equilibrium with positive firing costs studied in proposition 5 . They carry the same flavor, but a certain number of subtleties must be taken into account. 


\subsection{Some comparative statics results}

Before we discuss comparative statics results, it is useful to discuss two important details.

When parameters are held fixed, one can use either $F$ or $x$ as the policy variable over which people vote. Even though $F$ was the true institution over which people vote, we found it more convenient analytically to express utility as a function of $x$ and to maximize over that variable when determining the equilibrium. However, one has to be careful when doing comparative statics analysis because a change in parameters will typically also change the relationship between $x$ and $F$ as defined by (15). Intuitively, the true measure of job protection relevant to insiders is $x$; but it is achieved indirectly through an institution represented by $F$. A change in parameters may, for example, increase the equilibrium value of $x$ and at the same time alter the effect of $F$ on $x$ in such a way that despite the greater duration of jobs firing costs have actually decreased. Thus we shall be careful in distinguishing the effects of parameters on "job protection", as measured by $x$, and on firing costs, as measured by $F$.

The other problem is that parameters typically alter the steady-state unemployment rate and therefore the position of the median voter in the age distribution of firms. This effect is interesting in itself but one may also want to compare two economies that have different parameter values but would have the same unemployment rate in the original steady state; i.e., we also want to perform comparative statics holding the unemployment rate constant. As long as we consider a parameter other than $\delta$, this boils down to looking simply at the effects of parameter shifts on the position of the VV curve, ignoring shifts in the SS curve (since (24) tells us that given $x$, $z_{m}$ only depends on $u$ and $\left.\delta\right) .{ }^{23}$ One can always reintroduce the effect on

\footnotetext{
${ }^{23}$ Formally, to maintain unemployment constant in the original steady state when a parameter changes, one has to consider a simultaneous shift in that parameter and the
} 
unemployment by looking at the shift in the SS curve.

Because of these two issues, the analytical results we are able to establish are somewhat limited. They are summarized in proposition 6 .

\section{PROPOSITION $6-$}

(i) A marginal increase in $\varphi$ always shifts the VV curve to the right. Consequently, controlling for initial unemployment, the degree of job protection $x$ increases.

(ii) Assume that the equilibrium value of $x$ is such that

$$
x \leq \frac{r+\delta}{g(r+\delta-g)}
$$

Then, a marginal increase in $g$ always shifts the $V V$ curve to the left around that equilibrium. Consequently, controlling for initial unemployment, the degree of job protection $x$ decreases.

Proof - See Appendix.

Note that condition (26) is quite reasonable. Fog $r=5 \%, \delta=10 \%$, and $g=2 \%$ per year we get $x \leq 50 \times 15 \div 13=57.7$ years.

Proposition 6 confirms our intuition that a greater share in bargaining and slower growth increase the support for employment protection. However, the result is limited in the sense that it tells us that job protection moves in this way in terms of outcome, and given the unemployment rate. As we saw in proposition 2 , an increase in $\varphi$ also increases $x$ given $F$, so firing costs may well be reduced because the same value of the firing cost gives greater job protection. An increase in $g$ also reduces $x$ given $F$, so even though people want less job protection they may well end up voting for higher firing

parameter $b$, which has an impact on $\theta$ and $u$ but does not appear in the formulae (20) and (21) that represent the voters' utility functions. Thus one can always change $b$ in such a way that $u$ is unchanged, while the shift in VV is independent of the value of $b$. 
costs. Furthermore, both $\varphi$ and $g$ have an ambiguous impact on the position of the SS curve. ${ }^{24}$ Therefore, whereas controlling for unemployment the effect of these parameters on $x$ is unambiguous, it becomes ambiguous if one reintroduces the fact that they affect the equilibrium rate of unemployment.

\subsection{Numerical simulations.}

To complete our understanding of the model, here we present some numerical simulations. While the parameters we choose are realistic, this simulations are not meant to calibrate the model to actual data but to better understand it.

Table 1 reports the equilibrium values of $x, F, \theta$, and $u$ as a function of workers' bargaining power $\varphi$ for the following set of parameter values: $g=0.02, \delta=0.1, r=0.05, \rho=1 . b$ was set to -4 , which pins down the unemployment rate at $10 \%$ for $\varphi=0.5$ and no firing costs. The critical value of $\varphi$ for full flexibility to be an equilibrium is $r / g=0.4$. The rigid equilibrium of proposition 5 converges to full flexibility as $\varphi$ converges to 0.4 from above.

In all cases condition (23) was satisfied by a wide margin and equilibrium was unique, as VV was far steeper than SS.

Table 1 completes our knowledge of the dependence of $x$ and $F$ on $\varphi$ when we consider the shift in SS in addition to the shift in VV. The result that job protection $x$ increases with $\varphi$ is confirmed. In fact, $x$ is quite sensitive to $\varphi$. An increase in the workers' share by $10 \%$ lengthens plant duration by about 5 years. The response of the firing cost is hump-shaped. For $\varphi$ between 0.4 and 0.7 , it is increasing with $\varphi$, moving from 0 to $1 / 3,4$ months

\footnotetext{
${ }^{24} g$ has an ambiguous impact on $\theta$ and on unemployment. An increase in $\varphi$ increases $\theta$ and $u$, but at the same time the value of $F$ associated with a given value of $x$ is smaller when $\varphi$ is larger. As unemployment increases with $F$, this means that at around any point $(x, z)$ along the SS curve unemployment may either be higher or lower when $\varphi$ increases, so that we do not know where SS shifts.
} 
of a man's output in the most productive vintage. Beyond $\varphi=0.7 \mathrm{~F}$ declines as the direct effect of $\varphi$ on $x$ becomes stronger. Labor market tightness falls with $\varphi$, while unemployment rises with it. This is not surprising, but one should point out that our simulations imply that firing costs actually reduce unemployment ${ }^{25}$; so that when $\varphi$ rises, the induced increase in $F$ actually mitigates the rise in unemployment, although it reinforces the fall in $\theta$, i.e. the increase in unemployment duration.

Next, in table 2 we look at the dependence of employment protection on the growth rate $g$. We have assumed the same parameters as in table 1 , and $\varphi=0.6$. The critical value of the growth rate beyond which firing costs disappear is $g=\varphi r=0.03$.

Table 2 shows that job protection sharply falls with growth. The order of magnitude is 10 years per percentage point. The associated pattern of firing costs is hump-shaped. (15) implies that as growth goes to zero $x$ goes to infinity, regardless of the value of the firing cost. Thus for very low growth it is not surprising that the firing cost is low, since firms incur only small losses from keeping workers a very long time. Thus, the firing cost increases with $g$ for low growth rates but falls with it at larger growth rates. Note that unemployment increases with growth; this is also typically true if one holds the firing cost constant: the job destruction effect of growth tends to outweigh its job creation effect ${ }^{26}$.

Another parameter of interest is $\delta$, the exogenous rate of job destruction. While it was difficult to deal with it analytically, intuitively we expect the political support for firing costs to be lower with $\delta$. First, $\delta$ typically increases job destruction and thus unemployment, thus reducing the plant age of the median voter. This is further reinforced by the fact that the distribution of plant ages among the employed shifts to the left. Finally $\delta$ increases the

\footnotetext{
${ }^{25}$ This is also what Bentolila and Bertola (1990) in their numerical simulations.

${ }^{26}$ as in Aghion and Howitt (1994).
} 
burden of firing costs, which also reduces an employed's gain from employment protection (the terms in $-\delta \rho F /(r-g)$ in $(21)$ ). Table 3 confirms that intuition. Therefore, greater turnover reduces the political support for employment protection legislation - to the extent that this source of turnover is unaffected by firing costs.

\section{Conclusion}

This paper has analyzed the relationship between the functioning of the labor market and other characteristics of the economy, on the one hand, and the political support for employment protection. We have been able to derive a simple rule for full flexibility to be an equilibrium, showed the existence of political equilibria with positive firing costs if that rule is violated, and investigated analytically and numerically the properties of that equilibrium.

The key message of the paper is that employment protection is more likely to arise in economies with slow growth and bigger employee rents in wage formation. This finding is in accordance with the observation that such legislation is more important in Europe than in the U.S, and suggests that periods of high growth may be the appropriate time for increasing labor market flexibility.

The scope for further research is wide. First, there are many labor market institutions that can be studied from a political economy perspective. ${ }^{27}$ Second, the present model has ignored some determinants of employment protection legislation and could be extended to tackle these aspects. For example, we have assumed infinitely lived workers. Clearly, if one allowed for finite lives people's preferences for employment protection would depend on their age, in addition to the age of their match. Older workers would be less willing to become unemployed. Another possible extension is to allow

\footnotetext{
${ }^{27}$ See Saint-Paul (1996) for a discussion.
} 
for business cycles. An important aspect of the current debate over labor market reform in Europe is which phase of the business is most appropriate to implement it; the answer given by informal discussion is less than clear-cut so that more theoretical guidelines are needed..$^{28}$

\footnotetext{
${ }^{28}$ The interactions between the macroeconomic environment and reform incentives is discussed in Bean (1998) and Calmfors (1998). Elmeskov et al. (1998) discuss the experience of some specific countries.
} 


\section{References}

Acemoglu, D. and J. Robinson (1998), "Inefficient redistribution", mimeo, MIT,

Aghion, P. and P. Howitt, (1994) "Growth and unemployment", Review of Economic Studies

Bean, C. (1998) "The interaction of aggregate demand policies and labourmarket reform", Swedish Economic Policy Review

Bentolila, S. and G. Bertola (1990) "Firing costs and labor demand, how bad is Eurosclerosis", Review of Economic Studies

and G. Saint-Paul (1994) "A model of labor demand with linear adjustment costs", Labour Economics

Bertola, G. (1990), "Job security, employment, and wages", European Economic Review

—_ (1994), "Flexibility, Investment, and Growth", Journal of Monetary Economics

Burda, M. (1992) "A note on firing costs and severance benefits in equilibrium unemployment", Scandinavian Journal of Economics

Caballero, R. and M. Hammour (1994) "The cleansing effects of recessions", American Economic Review

and — (1996) "On the timing and efficiency of creative destruction", Quarterly Journal of Economics

Calmfors, L. (1998) "Unemployment, labour market reform, and monetary union", mimeo, IIES, Stockholm

Coe, D. and D. Snower (1997), "Policy complementarities: the case for fundamental labor market reform", IMF Staff papers

Elmeskov, J., J. Martin, and S. Scarpetta, (1998) "Key lessons for labour market reforms; evidence from OECD countries", Swedish Economic Policy Review 
Grüner, H.P. (1998) "Unemployment and labor market reform: a contract theoretic approach", mimeo, Bonn U., September 1998

Hassler, J., J.V. Rodriguez Mora, K. Storesletten, and F. Zilibotti (1998), "Political determination of unemployment benefits", mimeo, IIES, Stockholm

Hopenhayn, H. and R. Rogerson (1993) "Job turnover and policy evaluation: a general equilibrium analysis", Journal of Political Economy

Johansen, Leif (1959) "Substitution versus fixed production coefficients in the theory of economic growth: a synthesis", Econometrica

Krusell, P. and J.V. Rios-Rull (1994) "Vested interests in a positive theory of stagnation and growth", Review of Economic Studies

Lazear, E. (1990) "Job security provisions and employment", Quarterly Journal of Economics

Mortensen, D. and C. Pissarides (1994) "Job creation and Job destruction in the theory of unemployment", Review of Economic Studies

Robinson, J. (1997), "The dynamics of labor market institutions", mimeo, USC

Saint-Paul, G. (1993), "On the political economy of labor market flexibility", NBER Macroeconomics Annual (1996), "Exploring the political economy of labor market institutions", Economic Policy

Shapiro, C. and J. Stiglitz (1984) "Equilibrium unemployment as a worker's discipline device", American Economic Review

Wright, R. (1986), "The determination of unemployment benefits and the dynamics of voting", Journal of Public Economics 


\section{Appendix}

\section{A microeconomic foundation for (7): the stealing model}

The idea that workers can appropriate a fraction $\varphi$ of the present discounted value of the match's output on top of their alternative value can be obtained as the outcome of a version of the Shapiro and Stiglitz model of efficiency wages.

Assume the reward from misconduct is not sparing effort but getting access to a technology allowing to steal a fraction $\psi$ of the firm's output. This technology is "safe" in the sense that this stealing activity is not verifiable by a court. However, in the process of trying to gain access to that technology the worker may be caught, in which case he is fired. When trying to gain such access he has a constant probability of success equal to $p$ per unit of time, and a constant probability $q$ of being caught.

Let $V_{e}$ the value of being employed by the firm if one does not steal nor try to steal. The value of stealing $V_{S}(s, t)$ is solution to

$$
r V_{S}(s, t)=w(s, t)+\psi a_{s}+\delta\left(V_{u}(t)-V_{S}(s, t)\right)+\partial V_{T} / \partial t
$$

This means that the worker gets his regular wage and gets a fraction $\psi$ of the firm's output as long as he is employed. Taking differences with (9), noting that at $t=T(s) V_{e}=V_{S}=V_{u}$ and integrating we get that:

$$
V_{S}=V_{e}+\psi S(s, t)
$$

Let $V_{T}$ be the value of trying to access the stealing technology. The Bellman equation for $V_{T}$ is

$r V_{T}(s, t)=w(s, t)+(q+\delta)\left(V_{u}(t)-V_{T}(s, t)\right)+p\left(V_{S}(s, t)-V_{T}(s, t)\right)+\partial V_{T} / \partial t$ 
The efficiency wage is the one that deters from trying, that is that achieves $V_{T} \leq V_{e}$. Because of involuntary unemployment firms will pick up the lowest possible level, the one that achieves

$$
V_{e}=V_{T}
$$

. Confronting (27) and (28) with (9) we get the "No Trying Condition" (NTC)

$$
V_{e}=V_{u}+\frac{p \psi}{q} S(s, t)
$$

This is identical to $(7)$ with $\varphi=\frac{p \psi}{q}$. Note that one may have in principle $\varphi>1$, although for any $V_{u} \geq 0$ this would put the present discounted value of wages above that of output, thus deterring any entry.

\section{Proof of Proposition 1}

The economy may be in one of four regimes.

A. Regime 1 prevails when $x$ is finite and $\theta>0$. In that case both (12) and (13) hold with equality. One can rewrite (12) and (13) along a balanced growth path, yielding:

$$
\begin{aligned}
& 0=(1-\varphi) \frac{1-e^{-(r+\delta) x}}{r+\delta}-\theta \varphi \frac{1-e^{-(r+\delta) x}}{r+\delta} \frac{1-e^{-(r+\delta-g) x}}{r+\delta-g} \\
&-(b+\delta \rho F) \frac{1-e^{-(r+\delta-g) x}}{r+\delta-g}-F e^{-(r+\delta-g) x} \\
&(\varphi-1) e^{-g x}+b+\theta \varphi \frac{1-e^{-(r+\delta) x}}{r+\delta}=(r+\delta(1-\rho)-g) F
\end{aligned}
$$

Eliminating $\theta \varphi \frac{1-e^{-(r+\delta) x}}{r+\delta}$ between the two we get:

$$
(1-\varphi)\left[\frac{1}{r+\delta}+\frac{g e^{-(r+\delta) x}-(r+\delta) e^{-g x}}{(r+\delta)(r+\delta-g)}\right]=F,
$$

which is just (15). Note that the LHS is increasing in $x$, rising from 0 to $(1-\varphi) /(r+\delta)$ as $x$ rises from 0 to infinity. Consequently this will yield a finite value of $x$ if and only if $F<(1-\varphi) /(r+\delta)$. 
$\theta$ can then be computed by substituting $x$ in either (29) or (30), and by combining the two we get (16).

(16) yields a strictly positive value for $\theta$ if and only if

$$
\frac{g(1-\varphi)}{r+\delta}\left(1-e^{-(r+\delta) x}\right)<1-b-\varphi-\delta \rho F
$$

The LHS of that inequality is smaller than $\frac{g(1-\varphi)}{r+\delta}$. In the zone where $F<(1-\varphi) /(r+\delta)$, its RHS is greater than $1-b-\varphi-\delta \rho(1-\varphi) /(r+\delta)=$ $-b+(1-\varphi)(r+\delta(1-\rho)) /(r+\delta)$. Given assumption (14), this is greater than $g(1-\varphi) /(r+\delta)$.

Consequently, (31) always hold if $F<(1-\varphi) /(r+\delta)$ and if (14) holds. In this zone, (29)-(30) therefore always deliver a finite value of $x$ and a strictly positive value for $\theta$. This is an equilibrium. This proves statement 2 .

B. Regime 2. This is the regime where $x=+\infty$ and $\theta>0$. In this regime, the free-entry condition holds with equality but the optimal stopping condition is an inequality.

The free-entry condition can be obtained by letting $x$ go to infinity in (29). We get

$$
0=\frac{1-\varphi}{r+\delta}-\frac{\theta \varphi}{(r+\delta)(r+\delta-g)}-\frac{b+\delta \rho F}{r+\delta-g}
$$

The optimal stopping condition is obtained by writing that the LHS of (30) must be smaller than its RHS for $x=+\infty$ :

$$
b+\theta \varphi /(r+\delta) \leq(r+\delta(1-\rho)-g) F
$$

Eliminating $\theta$ between (32) and (33) we get that (33) may be replaced by $F \geq(1-\varphi) /(r+\delta)$. At the same time, for (32) to yield a positive value of $\theta$, it must be the case that $\frac{1-\varphi}{r+\delta}-\frac{b+\delta \rho F}{r+\delta-g}>0$, or equivalently $F<$ $\frac{1}{\delta \rho}\left[(1-\varphi)\left(\frac{r+\delta-g}{r+\delta}\right)-b\right]$.

Thus, for this regime to hold, it must be that $(1-\varphi) /(r+\delta) \leq F<$ $\frac{1}{\delta \rho}\left[(1-\varphi)\left(\frac{r+\delta-g}{r+\delta}\right)-b\right]$. Conversely, if this set of inequalities hold, then 
(32) yields a positive value of $\theta$ for which (33) is satisfied. So we have an equilibrium. Finally, assumption (14) guarantees that $(1-\varphi) /(r+\delta)<$ $\frac{1}{\delta \rho}\left[(1-\varphi)\left(\frac{r+\delta-g}{r+\delta}\right)-b\right]$. So this interval is non empty. This proves statement 3 .

C. Regime 3. This is the regime where $x<+\infty$ and $\theta=0$. It is now the free entry condition which is an inequality, while the optimal stopping condition is an equality. These two conditions are now given by

$$
\begin{gathered}
(\varphi-1) e^{-g x}+b=(r+\delta(1-\rho)-g) F \\
0 \geq(1-\varphi) \frac{1-e^{-(r+\delta) x}}{r+\delta}-(b+\delta \rho F) \frac{1-e^{-(r+\delta-g) x}}{r+\delta-g}-F e^{-(r+\delta-g) x}
\end{gathered}
$$

Eliminating $(b+\delta \rho F)$ between the two equations we find that we must have

$$
(1-\varphi)\left[\frac{1}{r+\delta}+\frac{g e^{-(r+\delta) x}-(r+\delta) e^{-g x}}{(r+\delta)(r+\delta-g)}\right] \leq F,
$$

While eliminating $b$ between (34) and (14) we get

$$
F \leq \frac{1-\varphi}{r+\delta}-\frac{(1-\varphi) e^{-g x}}{r+\delta(1-\rho)-g}
$$

(36) and (37) imply that

$$
\frac{g e^{-(r+\delta) x}}{(r+\delta)(r+\delta-g)} \leq \frac{e^{-g x}}{r+\delta-g}-\frac{e^{-g x}}{r+\delta(1-\rho)-g} \leq 0,
$$

which contradicts the assumption that $x$ is finite. Consequently, under (14) this regime never prevails in equilibrium.

D. Regime 4. This is when $x=+\infty$ and $\theta=0$. Both conditions are inequalities, so that we must have

$$
b \leq(r+\delta(1-\rho)-g) F
$$




$$
0 \geq \frac{1-\varphi}{r+\delta}-\frac{b+\delta \rho F}{r+\delta-g}
$$

Clearly, (39) implies

$$
F \geq \frac{1}{\delta \rho}\left[(1-\varphi)\left(\frac{r+\delta-g}{r+\delta}\right)-b\right]
$$

Assume now that this inequality holds and that (14) is satisfied. Then because of (14) we have both

$$
\frac{1}{\delta \rho}\left[(1-\varphi)\left(\frac{r+\delta-g}{r+\delta}\right)-b\right]>(1-\varphi) /(r+\delta)
$$

(40) and (41) imply $F \geq(1-\varphi) /(r+\delta)$. Substituting that into (14) we get (38). Therefore under (14), (40) is necessary and sufficient for regime 4 to prevail. This proves statement 4 .

Given that case A,B, and D span the whole set of possible values of $F$, we have proved statement 1 -a balanced growth path always exists.

\section{Proof of Proposition 2}

The dependence of $x$ on $F$ and $\varphi$ is trivially obtained from (15). Next, the derivative of the LHS of (15) with respect to $g$ has the same sign as $e^{-(r+\delta) x}+\left((x(r+\delta-g)-1) e^{-g x}\right.$, which is positive since $e^{-g x}-e^{-(r+\delta) x}<$ $x(r+\delta-g) e^{-g x}$ (because $\left.1-e^{-a}<a, \forall a>0\right)$. This implies $\partial x / \partial g<0$. Finally, the derivative of the LHS with respect to $r+\delta$ has the same sign as

$$
H(x ; r, g)=-(r-g)^{2}-r g(r-g) x e^{-r x}+r^{2} e^{-g x}-g(2 r-g) e^{-r x}
$$

, where we assume $\delta=0$ to save on notation. Now, one has $H(0 ; r, g)=0$ and $\partial H / \partial x=r^{2} g e^{-r x}\left[1-e^{(r-g) x}+(r-g) x\right]<0$ (because $\left.e^{a}>1+a, \forall a>0\right)$. Consequently $H(x ; r, g)<0 \forall x>0$. Therefore the LHS of (15) goes down with $r+\delta$,implying $\partial x / \partial(r+\delta)>0 . \square$ 


\section{Proof of Lemma 2}

To prove (i) just take the derivative of the RHS of (21) with respect to $z$. To prove (ii) and (iii), first note that (15) implies that

$$
\frac{d F}{d x}=\frac{\delta \rho}{r-g} \frac{(1-\varphi) g}{r+\delta-g}\left[e^{-g x}-e^{-(r+\delta) x}\right]
$$

Then take the derivative of the RHS of (21) with respect to $x$ and note that when multiplied by $e^{(r+\delta) x}$ it is proportional to

$$
\varphi e^{(r+\delta-g) z}-\frac{(1-\varphi) g}{r-g}-\frac{\delta \rho}{r-g} \frac{(1-\varphi) g}{r+\delta-g} e^{(r+\delta-g) x}-\frac{\delta \rho}{r-g} \frac{(1-\varphi) g}{r+\delta-g}
$$

which is strictly decreasing in $x$ and less than zero at $x=0$ if and only if $\varphi e^{(r+\delta-g) z}-\frac{(1-\varphi) g}{r-g} \leq 0$.

Finally, to prove (iv) note that this same derivative is increasing in $z . \square$

\section{Proof of proposition 4}

PROOF - Assume (22), or equivalently $\varphi(r-g) /((1-\varphi)(r-g)) \leq 1$ holds. Then, part (ii) of Lemma 2 implies that an employed's utility is decreasing in $x$ for $z=0$. Thus $V_{e}(z=0, x \geq 0) \leq V_{e}(z=0, x=0)=V_{u}(x=0)$. But, part (i) implies $V_{e}(z \geq 0, x) \leq V_{e}(z=0, x)$. Confronting these two inequalities we see that $V_{u}(x=0)=V_{e}(z, x=0)>V_{e}(z, x)$. Therefore any alternative to $x=0$ is defeated by unanimity. This proves (i) and sufficiency in (ii); to prove necessity, assume $F=0$ is a stationary equilibrium. Then the employed's distribution has a mass at $x=0$. To prevent $F=0$ from being defeated by a marginal increase in firing costs, it is necessary that $\partial V_{e} / \partial x \leq 0$ at $z=0$. But, according to part (ii) of the lemma, (22) must then hold. 


\section{Proof of proposition 5}

We are now in a position to derive a condition for a local political equilibrium to exist.

Assume people are initially distributed over $\left[0, x_{0}\right]$, with the steady state distribution of employment corresponding to $x_{0}$. Define as the median the value of $z, z_{m}$ such that

$$
(1-u) \int_{z_{m}}^{x_{0}} g(v) d v=1 / 2
$$

where $u$ and $g($.$) are the steady state unemployment level and employment$ density corresponding to $x=x_{0}$.

Our candidate equilibrium is the value of $x, x^{*}$, which maximizes $V_{e}\left(x, z_{m}\right)$ for $x \geq z_{m}$. Given our assumption that $\varphi>g / r$, such value is strictly greater than $z_{m}$ and satisfies $\partial V_{e}\left(x, z_{m}\right) / \partial x=0$. (See Lemma 2).

First, it is clear that $x^{*}$ defeats any increase in the firing cost, since one has $\partial V_{u} / \partial x<0$ (lemma 1) and $\partial V_{e}\left(x^{*}, z\right) / \partial x \leq 0$ for all $z \leq z_{m}$ (lemma 2). Hence at least $50 \%$ of the voters prefer $x^{*}$ to a marginally greater value.

Consider now a marginal reduction in the firing cost. We have to distinguish three cases.

1. $x^{*}>x_{0}$. In that case, nobody will lose one's job if the outcome if $x^{*}$ or a marginally different alternative. The relevant utility is given by $V_{e}(x, z)$ (which is single-peaked) for all employed workers. Given that $\partial V_{e}(x, z) / \partial x \geq$ 0 for all workers such that $z_{m} \leq z \leq x_{0}$, at least $50 \%$ of the electorate prefers $x^{*}$ to a marginally lower value. $x^{*}$ is then clearly an equilibrium.

2. $x^{*}<x_{0}$. In that case, all workers such that $z \geq x^{*}$ lose their jobs if the outcome is $x^{*}$ or a marginally smaller value $x^{\prime}$. Their utility is given by $V_{u}$, so that they all prefer a marginally smaller value. Let $z_{m}-\varepsilon$ denote a worker just indifferent between $x^{*}$ and $x^{\prime}$. Workers who prefer $x^{*}$ over $x^{\prime}$ are those such that $z_{m}-\varepsilon \leq z \leq x^{*}$, which is strictly below $50 \%$ of the people given that there is an infinitesimal mass of workers between $z_{m}-\varepsilon$ and $z_{m}$, 
and a non infinitesimal mass between $x^{*}$ and $x_{0}$.

Thus, $x^{*}$ is defeated by a marginal reduction in firing costs.

3. $x^{*}=x_{0}$. This is the most relevant case as it would correspond to a stationary equilibrium. In that case workers between $x^{\prime}$ and $x_{0}$ lose their jobs for $x=x^{\prime}$ but keep it for $x=x_{0}$. Let $x_{0}-\eta$ denote a worker just indifferent between being employed under $x_{0}$ and losing his job under $x^{\prime}$.

$$
V_{e}\left(x_{0}, x_{0}-\eta\right)=V_{u}\left(x^{\prime}\right)-k a_{t}\left(1-e^{-g\left(x_{0}-\eta\right)}\right)
$$

All workers such that $x_{0}-\eta \leq z \leq x_{0}$ favor $x^{\prime}$ over $x_{0}$. All workers such that $x^{\prime} \leq z \leq x_{0}-\eta$ favor $x_{0}$ over $x^{\prime}$.

Noting $x^{\prime}=x_{0}-\alpha, \alpha \ll 1$, we can rewrite the above equation as

$$
\eta=\alpha \frac{d V_{u} / d x\left(x_{0}\right)}{\partial V_{e} / \partial z\left(x_{0}, x_{0}\right)+a_{t} g k e^{-g x_{0}}}
$$

Let $z_{m}-\varepsilon$ denote again a worker just indifferent between $x_{0}$ and $x^{\prime}$, and who would keep his job under both alternatives:

$$
V_{e}\left(x_{0}, z_{m}-\varepsilon\right)=V_{e}\left(x^{\prime}, z_{m}-\varepsilon\right)=V_{e}\left(x_{0}-\alpha, z_{m}-\varepsilon\right)
$$

All workers such that $0 \leq z \leq z_{m}-\varepsilon$ favor $x^{\prime}$ over $x_{0}$. All workers such that $z_{m}-\varepsilon \leq z \leq x^{\prime}$ favor $x_{0}$ over $x^{\prime}$. Making a second order Taylor expansion of that formula and noting that $\partial V_{e}\left(x, z_{m}\right) / \partial x=0$ we get that

$$
\varepsilon=\alpha \frac{-\partial^{2} V_{e} / \partial x^{2}\left(x_{0}, z_{m}\right)}{2 \partial^{2} V_{e} / \partial x \partial z\left(x_{0}, z_{m}\right)}
$$

$x_{0}$ will defeat $x^{\prime}$ if and only if

$$
(1-u) \int_{z_{m}-\varepsilon}^{x_{0}-\eta} g(v) d v \geq 1 / 2
$$

which is equivalent to

$$
\eta \leq \varepsilon \frac{g\left(z_{m}\right)}{g\left(x_{0}\right)}=\varepsilon e^{\delta\left(x_{0}-z_{m}\right)}
$$


To compute $\varepsilon$ and $\eta$, just compute the relevant derivatives from (20) and (21), using (15). We get

$$
\begin{aligned}
\eta=\frac{1}{\varphi}[ & \left.\frac{\delta \rho(1-\varphi) g}{(r-g)(r+\delta-g)}\left(1-e^{-(r+\delta-g) x_{0}}\right)+\frac{g(1-\varphi)}{r-g} e^{-(r+\delta-g) x_{0}}\right] \\
\varepsilon= & -\frac{(1-\varphi)(r+\delta) g}{(r-g) \varphi(r+\delta-g)} e^{-(r+\delta-g) z_{m}} \\
& +\frac{\delta \rho g}{r-g} \frac{1-\varphi}{\varphi(r+\delta-g)^{2}}\left[(r+\delta) e^{-(r+\delta-g) z_{m}}-g e^{(r+\delta-g)\left(x_{0}-z_{m}\right)}\right] \\
& +\frac{r+\delta}{r+\delta-g}
\end{aligned}
$$

Let

$$
\begin{gathered}
A=\frac{\delta \rho g(1-\varphi)}{(r-g)(r+\delta-g)} \\
B=\frac{(1-\varphi) g}{r-g}
\end{gathered}
$$

Note that the condition $\partial V_{e}\left(x_{0}, z_{m}\right) / \partial x=0$ can be written as

$$
-A\left(e^{-g x}-e^{-(r+\delta) x}\right)-B e^{-(r+\delta) x}+\varphi e^{-g z_{m}} e^{-(r+\delta)\left(x-z_{m}\right)}=0
$$

Or, equivalently

$$
\varphi e^{(r+\delta+g) z_{m}}=\left(B+A\left(e^{(r+\delta-g) x_{0}}-1\right)\right)
$$

Substituting (50),(47), and (48) into (46) and (45) we get that

$$
\begin{gathered}
\varepsilon=\left[-B \frac{r+\delta}{r+\delta-g}+A \frac{r+\delta-g e^{(r+\delta-g) x_{0}}}{r+\delta-g}\right]\left[B+A\left(e^{(r+\delta-g) x_{0}}-1\right)\right]^{-1} \\
+\frac{r+\delta}{r+\delta-g} \\
\eta=\frac{1}{\varphi}\left[B e^{-(r+\delta-g) x_{0}}+A\left(1-e^{-(r+\delta-g) x_{0}}\right]\right.
\end{gathered}
$$


Consequently, (44) is equivalent to

$$
\begin{aligned}
& \frac{1}{\varphi}\left[B e^{-(r+\delta-g) x_{0}}+A\left(1-e^{-(r+\delta-g) x_{0}}\right]\left[B+A\left(e^{(r+\delta-g) x_{0}}-1\right)\right] \cdot e^{-\delta\left(x_{0}-z_{m}\right)}\right. \\
\leq & {\left[-B \frac{r+\delta}{r+\delta-g}+A \frac{r+\delta-g e^{(r+\delta-g) x_{0}}}{r+\delta-g}\right]+\frac{r+\delta}{r+\delta-g}\left[B+A\left(e^{(r+\delta-g) x_{0}}-1\right)\right] } \\
= & A e^{(r+\delta-g) x_{0}}
\end{aligned}
$$

Dividing both sides by $A e^{(r+\delta-g) x_{0}}$ and rearranging we see that this is equivalent to

$$
\begin{aligned}
& \frac{e^{-\delta\left(x_{0}-z_{m}\right)}}{\varphi}\left(A\left(1-2 e^{-(r+\delta-g) x_{0}}+e^{-2(r+\delta-g) x_{0}}\right)\right. \\
& +\frac{B^{2}}{A} e^{-2(r+\delta-g) x_{0}}+2 B\left(e^{-(r+\delta-g) x_{0}}-e^{-2(r+\delta-g) x_{0}}\right) \\
\leq & 1
\end{aligned}
$$

A sufficient condition for that to hold is

$$
\frac{A+B^{2} / A+2 B}{\varphi} \leq 1
$$

Plugging (47) and (48) we see that this is equivalent to

$$
\frac{(r+\delta(1-\rho)-g)^{2}}{\delta \rho(r+\delta-g)} \leq \frac{\varphi(r-g)}{g(1-\varphi-r k)}
$$

which is just (23).

Therefore, if $x^{*}>x_{0}$, it is an equilibrium; if $x^{*}=x_{0}$ and (23) holds, it is an equilibrium. If $x^{*}<x_{0}$ it is not an equilibrium.

Next, we prove that any local stationary equilibrium must be a median voter one. Consider a stationary local equilibrium. The outcome must be $x_{0}$. Let $z_{m}$ be the median as defined above. Assume $\partial V_{e}\left(x_{0}, z_{m}\right) / \partial x>0$. Then more than $50 \%$ of the people favor a marginal increase of firing costs over $x_{0}$. Consequently $x_{0}$ is not an equilibrium. Assume next $\partial V_{e}\left(x_{0}, z_{m}\right) / \partial x<0$. Then given that $z_{m}<x_{0}$, by continuity there exists $z^{\prime}$ such that $z_{m}<z^{\prime}<x_{0}$ 
and that $\partial V_{e} / \partial x<0$ for all $z \leq z^{\prime}$. All these agents, who are more than 50 $\%$ of the population, keep their jobs for a marginal decline in firing costs and are made strictly better-off by such a move.

The last thing that remain to be proved is that there actually exists an initial steady state such that $x^{*}=x_{0}$. As discussed in the text, this amounts to showing that VV and SS actually cross, i.e. that there exists a pair $\left(x_{0}, z_{m}\right)$ such that (43) (which defines SS) and (49) (which defines VV) simultaneously hold. The properties of SS are established in the text. As for VV, by multiplying (49) by $e^{g x}$ and letting $x$ go to infinity, we see that the terms in $e^{(g-r-\delta) x}$ become negligible, so that the relationship between $x$ and $z$ converges to:

$$
-A+\varphi e^{-(r+\delta-g)(x-z)} \approx 0
$$

Or equivalently

$$
z=x+\frac{\ln \frac{A}{\varphi}}{r+\delta-g}
$$

This proves that along $\mathrm{VV} z$ goes to infinity as $x$ does, and that it has an asymptote parallel to the 45 degree line. Consequently, it is above SS for high values of $x$.

Next, multiplying (49) by $e^{(r+\delta) x}$ and letting $x$ go to zero allow us to compute the intercept of VV:

$$
-B+\varphi e^{(r+\delta-g) z}=0
$$

Given that the condition $\varphi>g / \dot{r}$ is equivalent to $\varphi>B$, for that to hold one must have $e^{(r+\delta-g) z}<1$, i.e. $z<0$. This proves that the intercept of $\mathrm{VV}$ is negative, and therefore that it is below SS for low values of $x . \square$

\section{Proof of Proposition 6}


Differentiating (50) with respect to $x$ and $\varphi$ yields

$$
\begin{aligned}
d x\left\{\begin{array}{c}
-\frac{(1-\varphi) g(r+\delta)}{r-g} e^{-(r+\delta) x}+(r+\delta) \varphi e^{-g z} e^{-(r+\delta)(x-z)} \\
+\delta \rho \frac{(1-\varphi) g}{(r-g)(r+\delta-g)}\left[(r+\delta) e^{-(r+\delta) x}-g e^{-g x}\right]
\end{array}\right\} \\
=d \varphi\left\{\begin{array}{c}
\frac{g}{r-g} e^{-(r+\delta) x}+e^{-g z} e^{-(r+\delta)(x-z)} \\
+\delta \rho \frac{g}{(r-g)(r+\delta-g)}\left[e^{-g x}-e^{-(r+\delta) x}\right]
\end{array}\right\}
\end{aligned}
$$

Making use once more of (50) one can show that the term multiplying $d x$ is equal to

$$
C=(r+\delta-g) e^{-(r+\delta) x}\left[\varphi e^{(r+\delta-g) z}-\frac{(1-\varphi) g}{r-g}+\frac{\delta \rho(1-\varphi) g}{(r-g)(r+\delta-g)}\right],
$$

which is positive as long as $\varphi \geq g / r$.

The term multiplying $d \varphi$ is obviously positive. This proves that $x$ increases given $z$ in $(50)$ when $\varphi$ increases, in other words that the $\mathrm{VV}$ curve shifts to the right. This proves the first part of prop. 8 .

To prove the second part, differentiate now (50) with respect to $x$ and $g$ to get

$$
C . d x=D . d g
$$

where

$$
\begin{aligned}
D= & -e^{-(r+\delta) x}(1-\varphi) \frac{r}{(r-g)^{2}}-\varphi z e^{-g z} e^{-(r+\delta)(x-z)} \\
& -\delta \rho \frac{(1-\varphi)(r+\delta)}{(r-g)(r+\delta-g)^{2}}\left[e^{-g x}-e^{-(r+\delta) x}\right] \\
& +\delta \rho \frac{(1-\varphi) g}{(r-g)(r+\delta-g)} x e^{-g x}
\end{aligned}
$$

Assume, as in the text, that

$$
x \leq \frac{r+\delta}{g(r+\delta-g)}
$$

This clearly implies that

$$
\delta \rho \frac{(1-\varphi) g}{(r-g)(r+\delta-g)} x e^{-g x} \leq \delta \rho \frac{(1-\varphi)(r+\delta)}{(r-g)(r+\delta-g)^{2}} e^{-g x}
$$


And therefore that:

$$
\begin{aligned}
D \leq- & e^{-(r+\delta) x}(1-\varphi) \frac{r}{(r-g)^{2}}-\varphi z e^{-g z} e^{-(r+\delta)(x-z)} \\
& +\delta \rho \frac{(1-\varphi)(r+\delta)}{(r-g)(r+\delta-g)^{2}} e^{-(r+\delta) x}
\end{aligned}
$$

Finally, note that if

$$
1 \leq \frac{r(r+\delta-g)^{2}}{\delta(r-g)(r+\delta)}
$$

then, as $\rho<1$,

$$
\delta \rho \frac{(1-\varphi)(r+\delta)}{(r-g)(r+\delta-g)^{2}} e^{-(r+\delta) x} \leq e^{-(r+\delta) x}(1-\varphi) \frac{r}{(r-g)^{2}},
$$

which would imply $D<0$. Therefore, the only thing one has to show is (51). To prove it, develop the numerator and the denominator and get the difference between the two to get

$$
r^{3}+r g^{2}+r^{2} \delta-r \delta g+\delta^{2} g-2 r^{2} g
$$

This can be rewritten as

$$
r(r-g)^{2}+r \delta(r-g)+\delta^{2} g
$$

which is positive since $r>g$. This completes the proof of proposition 6 . 


\begin{tabular}{lllll}
\hline \hline$\varphi$ & $x$ (years) & $F$ & $\theta$ (per year) & $u(\%)$ \\
\hline 0.4 & 0 & 0 & $\infty$ & 8.0 \\
0.5 & $63 / 4$ & 0.16 & 2.09 & 8.9 \\
0.6 & $111 / 4$ & 0.286 & 1.32 & 10.0 \\
0.7 & $151 / 2$ & 0.337 & 1.0 & 11.2 \\
0.8 & $201 / 4$ & 0.317 & 0.815 & 12.4 \\
0.9 & 27 & 0.22 & 0.689 & 13.4 \\
\hline
\end{tabular}

Table 1: Effect of worker's bargaining power on firing costs and job protection. $g=2 \%, r=5 \%, \delta=10 \%, \rho=1, b=-4$.

\begin{tabular}{lllll}
\hline \hline$g(\%)$ & $x$ (years) & $F$ & $\theta$ (per year) & $u(\%)$ \\
\hline 0.5 & $253 / 4$ & 0.24 & 1.11 & 8.8 \\
1 & 20 & 0.336 & 1.14 & 9.1 \\
1.5 & $151 / 2$ & 0.347 & 1.2 & 9.6 \\
2 & $111 / 4$ & 0.285 & 1.33 & 10.0 \\
2.5 & $61 / 2$ & 0.147 & 1.74 & 10.7 \\
3 & 0 & 0 & $\infty$ & 12.0 \\
\hline
\end{tabular}

Table 2: Effect of the growth rate on firing costs and job protection. $\varphi=0.6, r=5 \%, \delta=10 \%, \rho=1, b=-4$.

\begin{tabular}{lllll}
\hline \hline$\delta(\%)$ & $x$ (years) & $F$ & $\theta($ per year $)$ & $u(\%)$ \\
\hline 1 & $841 / 2$ & 4.85 & 0.42 & 3.9 \\
3 & 35 & 1.79 & 0.6 & 7.1 \\
5 & 22 & 0.89 & 0.8 & 8.5 \\
10 & $111 / 4$ & 0.285 & 1.33 & 10.0 \\
15 & $71 / 2$ & 0.137 & 1.87 & 10.6 \\
20 & $53 / 4$ & 0.082 & 2.38 & 10.9 \\
40 & $23 / 4$ & 0.02 & 4.62 & 11.5 \\
\hline
\end{tabular}

Table 3: Effect of exogenous destruction on firing costs and job protection. $\varphi=0.6, r=5 \%, g=2 \%, \rho=1, b=-4$ 
Figure 1: preferences according to plant age and employment status

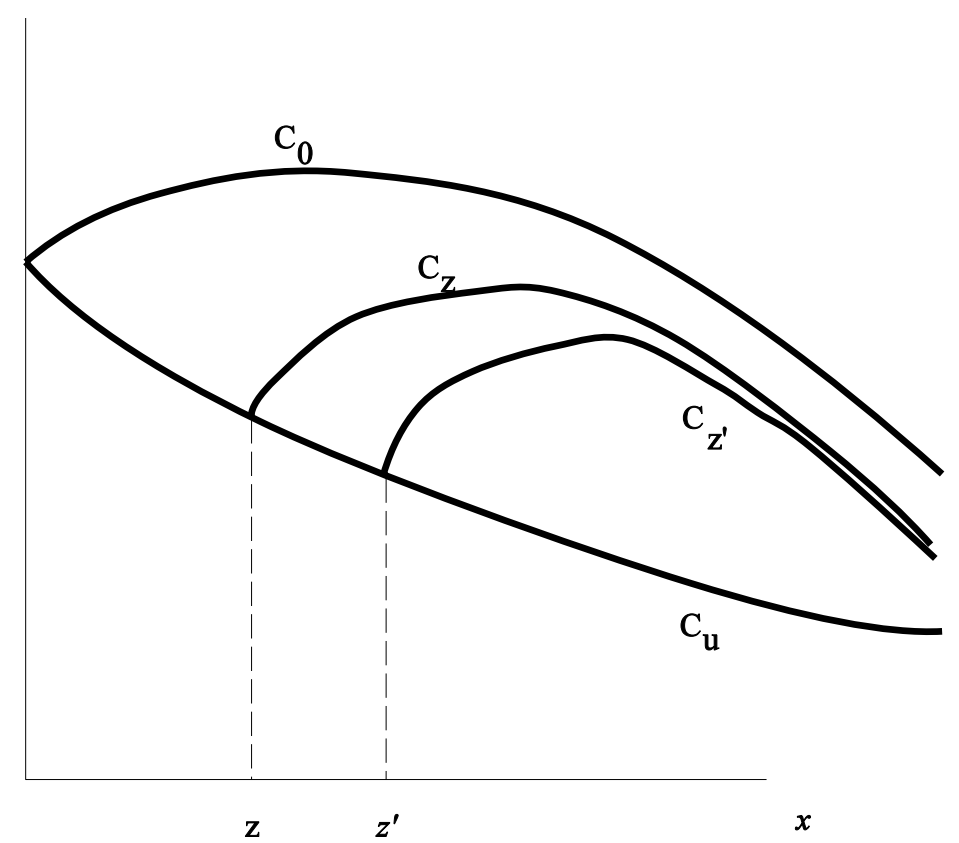


Figure $2 \mathrm{~b}$ : Voting on a reduction in firing costs

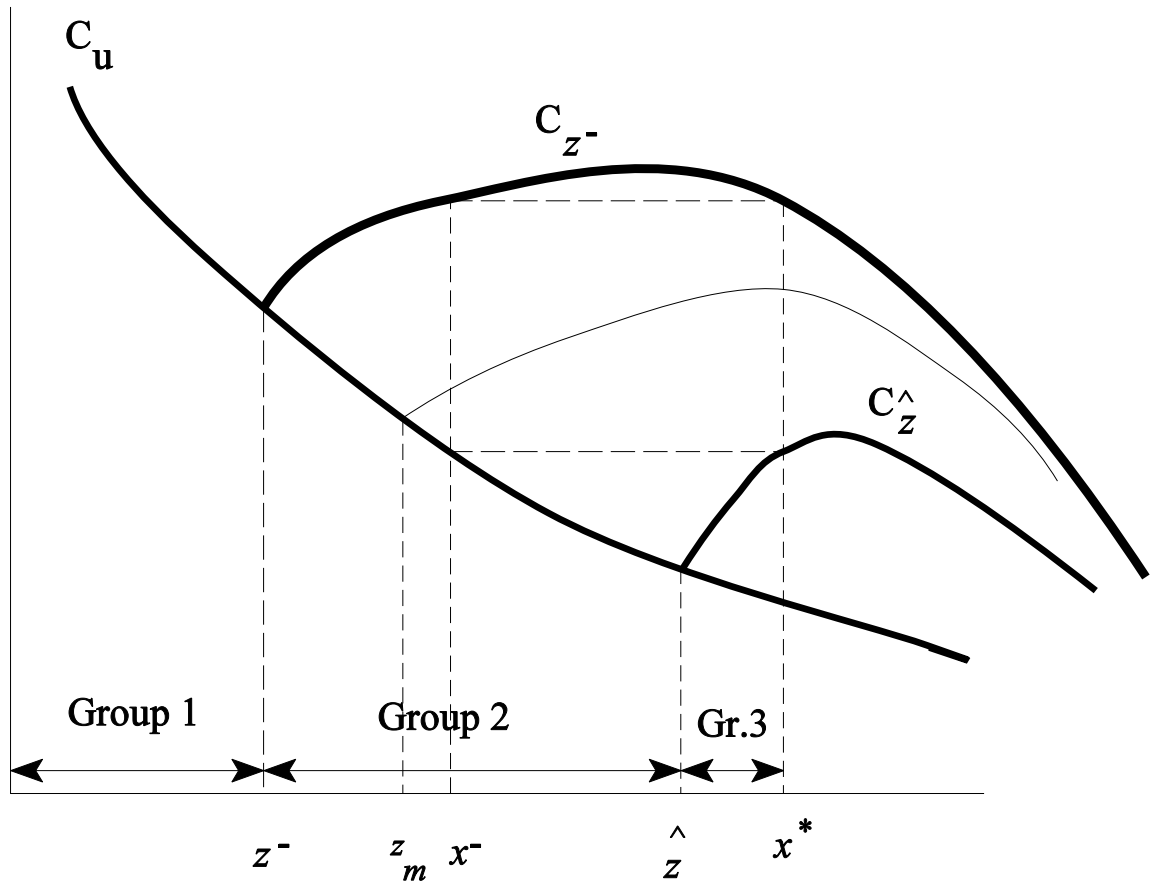


Figure 2a: Increase in firing costs

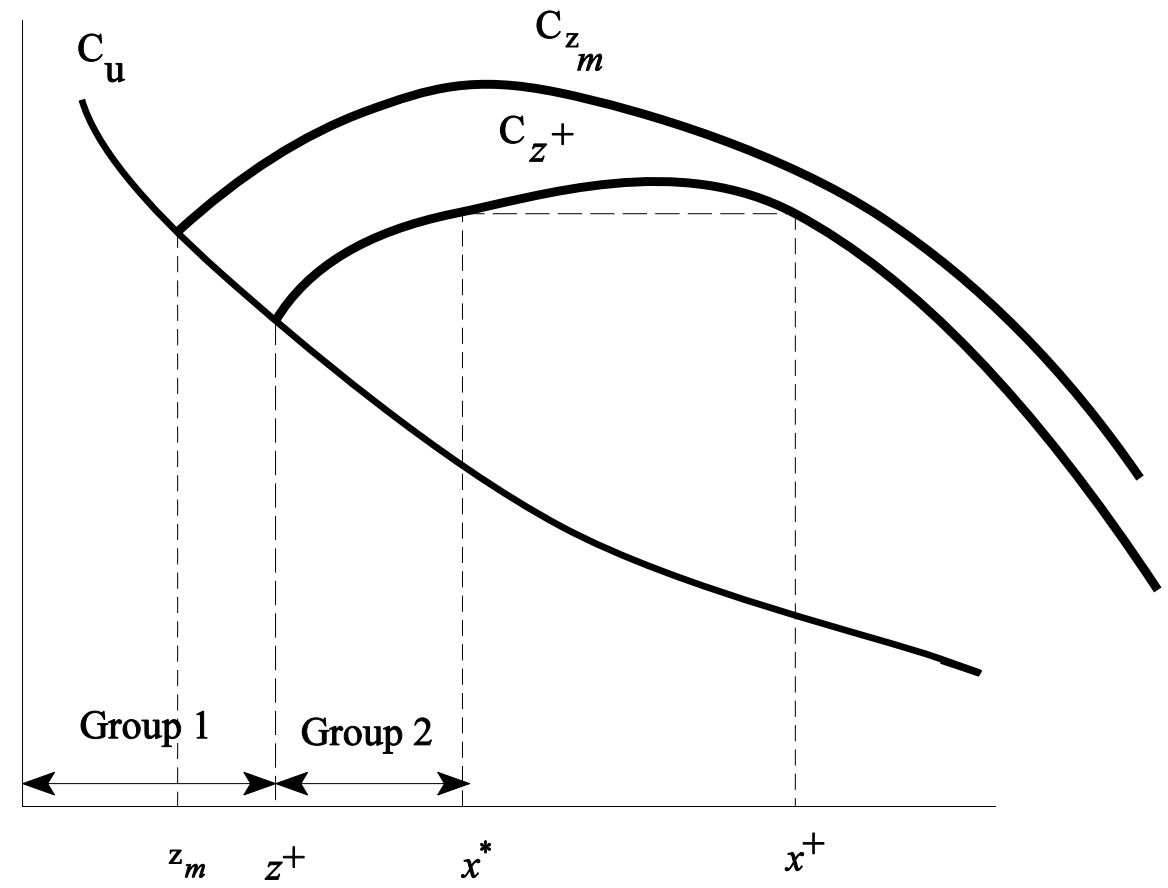


Figure 3: Condorcet Cycles

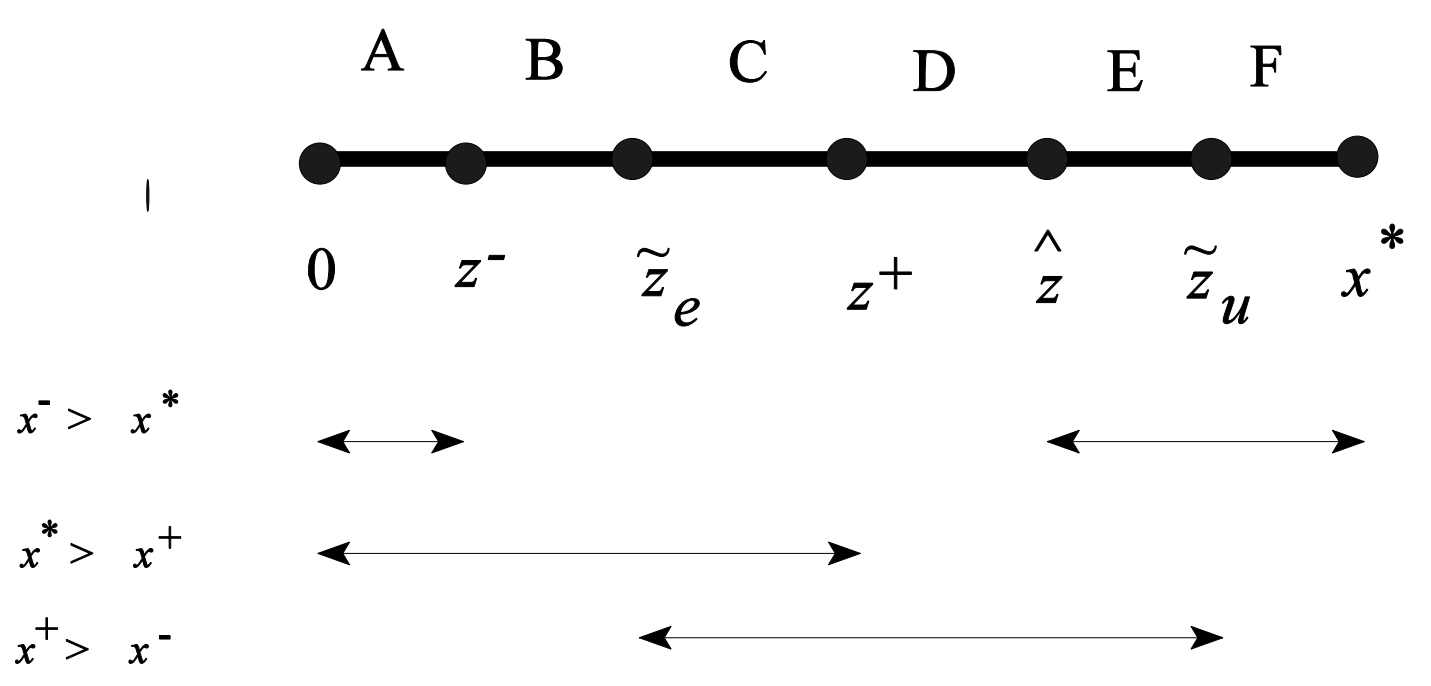


Figure 4: $F=x=0$ is a majority winner

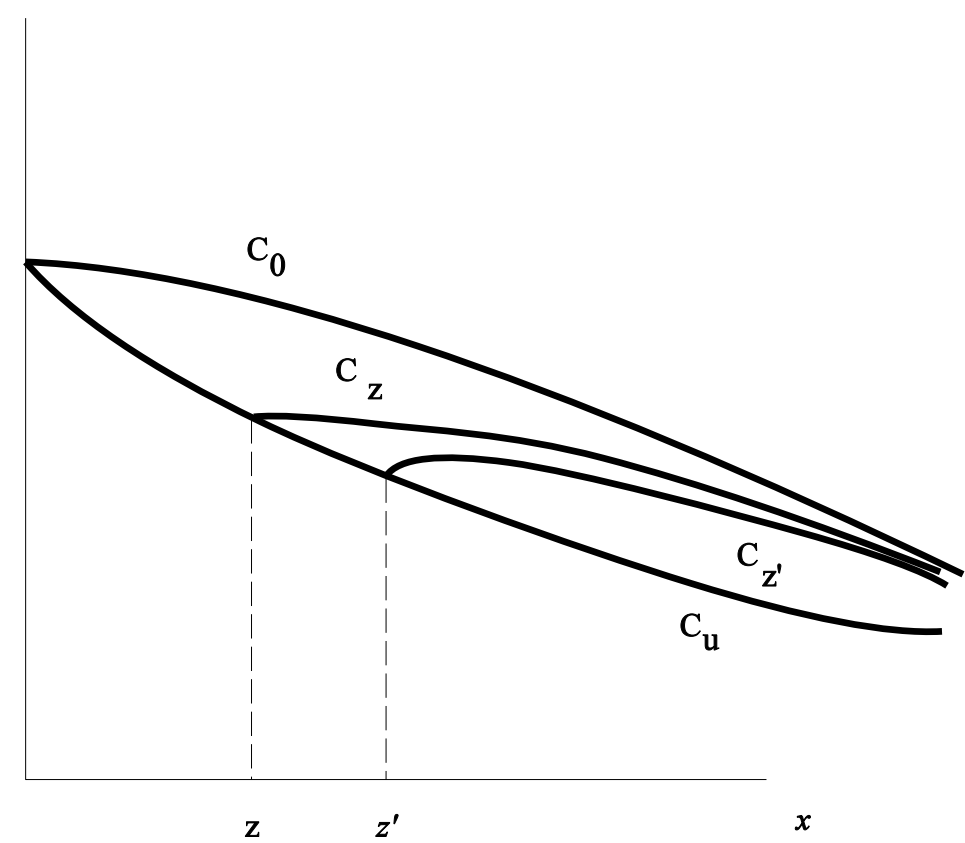


Figure 5: Political equilibrium determination

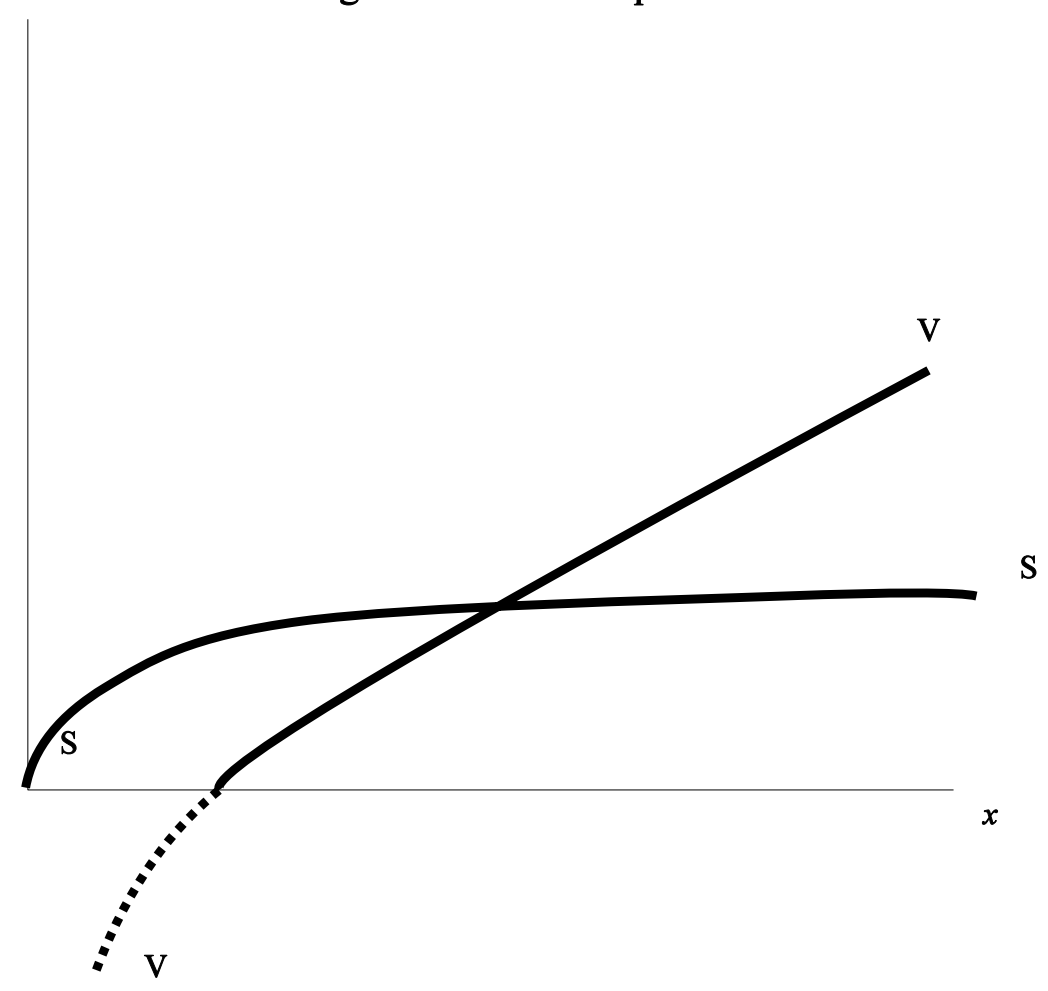


